Punjab University Journal of Mathematics (2021),53(11),793-811

https://doi.org/10.52280/pujm.2021.531103

\title{
Generalized Geometry of Tangential and Affine Configuration Chain Complexes
}

\author{
M. Khalid ${ }^{a, *}$, Mariam Sultana ${ }^{b}$, Azhar Iqbal ${ }^{c}$ and Javed $\operatorname{Khan}^{d}$ \\ ${ }_{a, b, d}$ Department of Mathematical Sciences, \\ Federal Urdu University of Arts, Sciences \& Technology, Karachi-75300, Pakistan, \\ ${ }^{c}$ Department of Basic Sciences, \\ Dawood University of Engineering \& Technology, Karachi-75800, Pakistan. \\ Corresponding author Email: ${ }^{a, *}$ khalidsiddiqui@ fuuast.edu.pk. \\ ${ }^{b}$ marium.sultana@fuuast.edu.pk, ${ }^{c}$ javid.afridi@ fuuast.edu.pk ${ }^{d}$ azhar.iqbal@duet.edu.pk
}

Received: 13 February, 2020 / Accepted: 23 October, 2021 / Published online: 25 November, 2021

\begin{abstract}
In this work, geometry of tangential and affine configuration chain complex is proposed in generalized form. Initially tangential and affine configuration chain complexes are connected for special case $n=6$, lastly this concept is generalized for any case $n \in \mathbb{N}$ through two types of generalized homomorphisms.
\end{abstract}

\section{AMS Subject Classification Codes: 11G55; 14F42; 19E15; 14R10}

Key Words: Tangent groups, Affine configuration complexes, Polylogarithms groups, Truncated Polynomial.

\section{INTRODUCTION}

The theory of affine configuration chain complexes is important for algebraic K-theory and algebraic geometry, first introduced by Suslin [23]. Afterwards, this theory was more developed by Khalid et al. $[14,15]$ and it has found many applications in different fields such as quantum physics, K-group theory, geometry and manifold theory. Initially, Polylogarithmic function

$$
L i_{p}(z)=\sum_{n=1}^{\infty} \frac{z^{n}}{n^{p}}, \quad z \leq 1
$$

was introduced by Leibniz with formula $\log (x y)-\log (x)-\log (y)=0$. Bloch introduced weight 1 poly-logarithmic groups $\mathcal{B}_{1}(F)$ by taking quotient of Z-module $Z\left[\mathbf{P}_{F}^{1} /\{0,1, \infty\}\right]$ and subgroup of Z-module, generated by the three term relation $[x]+[y]-[x y]$ [1]. Bloch also defined di-logarithmic group $\mathcal{B}_{2}(F)$ as the quotient of $Z\left[\mathbf{P}_{F}^{1} /\{0,1, \infty\}\right]$ by the subgroup generated by the five term relation cross ratio of four points $\sum_{i=0}^{4}(-1)^{i} r\left(v_{0}, \ldots, \hat{v}_{i}\right.$, 
$\left.\ldots, v_{4}\right)$. Goncharov [6-9] defined classical polylog group for weight 3 and then he generalized Bloch group for weight $n$ to define his own classical polylogarithmic generalized chain complex.

Goncharov [7] proposed the geometry of Bloch Suslin polylogarithmic and configuration chain complexes for weight 2 . Goncharov also introduced geometry between configuration and classical polylogarithmic chain complexes for weight $n=3$. Khalid et al. [12-19] not only redefine geometry for weight 2 and 3 but also generalize geometry for any weight $n \in \mathbb{N}$. Cathelineau [2-4] used derivation to introduce variant of Goncharov polylog chain complex in two different ways, first one was infinitesimal while other was tangential setting. Siddiqui [21] defined cross ratio of four points and the Siegels cross ratio property in tangential form and connected affine configuration and first order tangential chain complexes up to weight 3 . Hussain [11] defined second order tangent group and introduced geometry for weight 2 and 3. Khalid et al. [20] extended the work of Siddiqui to define geometry between tangential and affine configuration chain complexes up to weight 5 .

Present research work aims to extend the work of Khalid et al. [20] to propose generalized geometry between affine configuration and tangent group chain complexes. Section 2 is about background and basic ideas of affine configuration chain complexes, truncated polynomial and its rings, cross ratio and duel numbers cross ratio, classical polylogarithmic groups and its complexes, Tangent group and its generalized chain complex, geometry tangential and affine configuration chain complexes for weight 2 up to weigh 5. Section 3 describes the geometry of tangential and affine configuration complexes for weight 5 and last part of section 3 is generalization of the previous work. Section 4 is conclusion of the whole research work.

\section{BACKGROUND \& LITERATURE REVIEW}

2.1. Configuration Spaces and its Chain Complex. Let us assume that the general linear group of order $n$ is denoted by $G L_{n}(F)$, it is acting diagonally on some set $V^{n}$. The group action of $G L_{n}(F) * V^{n}=V^{n}$ produce $\left(v_{0}, \ldots, v_{n}\right)$ elements [21]. These elements are called configurations of an n-dimensional vector space $V^{n}$ defined over number field $F$. Let us consider a free abelian group $G_{n}(V)$ generated by the projective configuration of $\left(v_{1}, \ldots, v_{n}\right)$ points. Following maps are two types of differential morphism, first one is $d$ defined as

$$
d\left(v_{0}, \ldots, v_{n}\right) \rightarrow \sum_{i}^{n}(-1)^{i}\left(v_{0}, \ldots, \hat{v}_{i}, \ldots, v_{n}\right),
$$

$\hat{v}_{i}$ is a leaving out element and other differential map $p$ is defined as

$$
p\left(v_{0}, \ldots, v_{n}\right) \rightarrow \sum_{i}^{n}(-1)^{i}\left(v_{i} \mid v_{0}, \ldots, \hat{v}_{i}, \ldots, v_{n}\right)
$$


In [23] free abelian groups are connected using morphisms to get following configuration chain complex

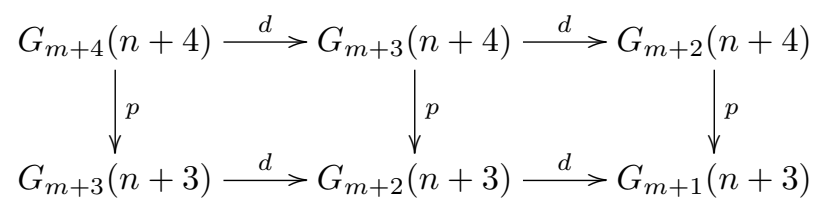

The chain complex is a bi-complex and each square in this chain is commutative (see [23]).

2.2. Affine Configuration Spaces. Assume that $\mathrm{F}$ is a field with characteristic 0 and the ring of $k^{t h}$ truncated polynomial is denoted by $F[\varepsilon]_{k}:=F[\varepsilon] / \varepsilon^{k}, k \geq 1$. Affine space $\mathbb{A}_{F[\varepsilon]_{n}}^{n}$ is a space defined over the truncated polynomial $F[\varepsilon]_{n}$.

Let $v=\left(c_{1}, c_{2}, c_{3}, \ldots, c_{n}\right)^{t} \in \mathbb{A}_{F}^{n} \backslash(0,0,0, \ldots, 0)^{t}$ and $v_{\varepsilon}=\left(c_{1, \varepsilon}, \ldots, c_{n, \varepsilon}\right)^{t} \in \mathbb{A}_{F}^{n}$ also $v_{\varepsilon^{n}}=\left(c_{1, \varepsilon^{k-1}}, c_{2, \varepsilon^{k-1}}, c_{3, \varepsilon^{k-1}}, \ldots, c_{n, \varepsilon^{k-1}}\right)^{t} \in \mathbb{A}_{F}^{n}[11]$. Assume that a free abelian $G_{m}\left(\mathbb{A}_{F[\varepsilon]_{k}}^{n}\right)$ generated by vectors $\left(v_{1}^{*}, \ldots, v_{m}^{*}\right)$ in affine space $\left(\mathbb{A}_{F[\varepsilon]_{k}}^{n}\right)$ [11] such that the vectors are defined as $v^{*}=v+v_{\varepsilon} \varepsilon+\ldots+v_{\varepsilon^{k-1}} \varepsilon^{k-1}$. Now we define the following boundary differential maps

$$
d: G_{m}\left(\mathbb{A}_{F[\varepsilon]_{k}}^{n}\right) \rightarrow G_{m-1}\left(\mathbb{A}_{F[\varepsilon]_{k}}^{n}\right)
$$

and

$$
p: G_{m}\left(\mathbb{A}_{F[\varepsilon]_{k}}^{n}\right) \rightarrow G_{m-1}\left(\mathbb{A}_{F[\varepsilon]_{k}}^{n-1}\right)
$$

by using above two maps following generalized affine configuration chain complex is constructed.

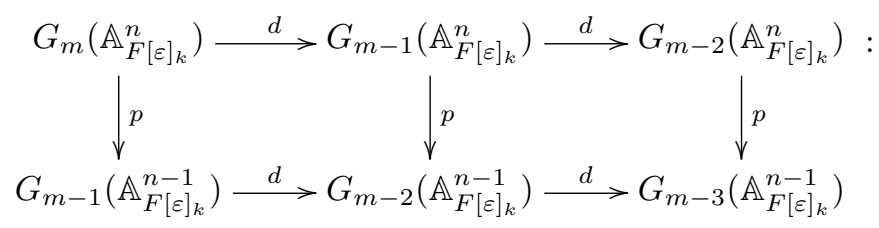

2.3. Cross Ratio and Siegel Cross Ratio Property of Four Points. Let $\left(v_{0}, \ldots, v_{3}\right)$ are four points then its cross ratio is defined as

$$
r\left(v_{0}, v_{1}, v_{2}, v_{3}\right)=\frac{\triangle\left(v_{0}, v_{3}\right) \triangle\left(v_{1}, v_{2}\right)}{\triangle\left(v_{0}, v_{2}\right) \triangle\left(v_{1}, v_{3}\right)}
$$

where $\left(v_{0}, \ldots, v_{3}\right) \in A_{F}^{2}$. Siegel [22] defined the following property of cross ratio

$$
1=\frac{\triangle\left(v_{0}, v_{3}\right) \triangle\left(v_{1}, v_{2}\right)}{\triangle\left(v_{0}, v_{2}\right) \triangle\left(v_{1}, v_{3}\right)}+\frac{\triangle\left(v_{0}, v_{1}\right) \triangle\left(v_{2}, v_{3}\right)}{\triangle\left(v_{0}, v_{2}\right) \triangle\left(v_{1}, v_{3}\right)} .
$$

2.3.1. Cross Ratio in a Polynomial Ring $F[\varepsilon]_{k}$. First we introduce the following cases

Case 1 : $k=1$ and $n=2$,

$$
\triangle\left(v_{1}^{*}, v_{2}^{*}\right)=\triangle\left(v_{1}^{*}, v_{2}^{*}\right)_{\varepsilon^{0}}=\triangle\left(v_{1}, v_{2}\right)
$$


Case 2: $\mathrm{k}=2$ and $\mathrm{n}=2$,

$$
\begin{aligned}
& \triangle\left(v_{1}^{*}, v_{2}^{*}\right)=\triangle\left(v_{1}^{*}, v_{2}^{*}\right)_{\varepsilon^{0}}+\triangle\left(v_{1}^{*}, v_{2}^{*}\right)_{\varepsilon^{1} \varepsilon} \\
& \triangle\left(v_{1}^{*}, v_{2}^{*}\right)_{\varepsilon^{1}}=\triangle\left(v_{1}, v_{2, \varepsilon}\right)+\triangle\left(v_{1, \varepsilon}, v_{2}\right)
\end{aligned}
$$

Case 3 : $\mathrm{k}=3$ and $\mathrm{n}=2$,

$$
\triangle\left(v_{1}^{*}, v_{2}^{*}\right)=\triangle\left(v_{1}^{*}, v_{2}^{*}\right)_{\varepsilon^{0}}+\triangle\left(v_{1}^{*}, v_{2}^{*}\right)_{\varepsilon^{1}} \varepsilon+\triangle\left(v_{1}^{*}, v_{2}^{*}\right)_{\varepsilon^{2}} \varepsilon^{2}
$$

where $\triangle\left(v_{1}^{*}, v_{2}^{*}\right)_{\varepsilon^{2}}=\triangle\left(v_{1}, v_{2, \varepsilon^{2}}\right)+\triangle\left(v_{1, \varepsilon}, v_{2, \varepsilon}\right)+\triangle\left(v_{1, \varepsilon^{2}}, v_{2}\right)$.

From [21] and [11] following are cross ratio relations in $F[\varepsilon]_{k}$

$$
\mathbf{r}\left(v_{0}^{*}, \ldots, v_{3}^{*}\right)=\left(r_{\varepsilon^{0}}+r_{\varepsilon^{1}} \varepsilon+\ldots+r_{\varepsilon^{k-1}} \varepsilon^{k-1}\right)\left(v_{0}^{*}, \ldots, v_{3}^{*}\right)
$$

where

$$
\begin{gathered}
r_{\varepsilon^{0}}\left(v_{0}^{*}, \ldots, v_{3}^{*}\right)=r\left(v_{0}, \ldots, v_{3}\right)=\frac{\triangle\left(v_{0}, v_{3}\right) \triangle\left(v_{1}, v_{2}\right)}{\triangle\left(v_{0}, v_{2}\right) \triangle\left(v_{1}, v_{3}\right)} \\
r_{\varepsilon^{1}}\left(v_{0}^{*}, \ldots, v_{3}^{*}\right)=\frac{\left\{\triangle\left(v_{0}^{*}, v_{3}^{*}\right) \triangle\left(v_{1}^{*}, v_{2}^{*}\right)\right\}_{\varepsilon}}{\triangle\left(v_{0}, v_{2}\right) \triangle\left(v_{1}, v_{3}\right)}-r\left(v_{0}, \ldots, v_{3}\right) \frac{\left\{\triangle\left(v_{0}^{*}, v_{2}^{*}\right) \triangle\left(v_{1}^{*}, v_{3}^{*}\right)\right\}_{\varepsilon}}{\triangle\left(v_{0}, v_{2}\right) \triangle\left(v_{1}, v_{3}\right)} \\
r_{\varepsilon^{2}}\left(v_{0}^{*}, \ldots, v_{3}^{*}\right)= \\
\frac{\left\{\triangle\left(v_{0}^{*}, v_{3}^{*}\right) \triangle\left(v_{1}^{*}, v_{2}^{*}\right)\right\}_{\varepsilon}}{\triangle\left(v_{0}, v_{2}\right) \triangle\left(v_{1}, v_{3}\right)}-r\left(v_{0}^{*}, \ldots, v_{3}^{*}\right) \frac{\left\{\triangle\left(v_{0}^{*}, v_{2}^{*}\right) \triangle\left(v_{1}^{*}, v_{3}^{*}\right)\right\}_{\varepsilon}}{\triangle\left(v_{0}, v_{2}\right) \triangle\left(v_{1}, v_{3}\right)}- \\
r\left(v_{0}, \ldots, v_{3}\right) \frac{\left\{\triangle\left(v_{0}^{*}, v_{2}^{*}\right) \triangle\left(v_{1}^{*}, v_{3}^{*}\right)\right\}_{\varepsilon}}{\triangle\left(v_{0}, v_{2}\right) \triangle\left(v_{1}, v_{3}\right)}
\end{gathered}
$$

and so on up to $r_{\varepsilon^{k-1}}$.

2.4. Classical Polylogarithmic Group Chain Complexes. Leibniz defined p-logarithm function by an absolutely convergent series

$$
\operatorname{Li}_{p}(z)=\sum_{n=1}^{\infty} \frac{z^{n}}{n^{p}}, \quad z \leq 1 .
$$

Let $Z\left[\mathbf{P}_{F}^{1} /\{0,1, \infty\}\right]$ be a free abelian group of $Z$-module generated by element [x] and defined over doubly punctured set.

2.4.1. Scissor Congruence Group. The classical polylogarithmic group $\mathcal{B}(F)$ is a quotient of $Z\left[\mathbf{P}_{F}^{1} /\{0,1, \infty\}\right]$ by Abel relation $\left[v_{1}\right]-\left[v_{2}\right]+\left[\frac{v_{2}}{v_{1}}\right]-\left[\frac{1-v_{2}^{-1}}{1-v_{1}^{-1}}\right]+\left[\frac{1-v_{2}}{1-v_{1}}\right], v_{1} \neq v_{2}$ and $v_{1}, v_{2} \neq 0,1[5,10]$.

2.4.2. Polylog Bloch Group for Weight-1. In [1] Bloch introduced a subgroup $\mathrm{R}_{1}(F) \subset$ $Z\left[\mathbf{P}_{F}^{1} /\{0,1, \infty\}\right]$, generated by the relation $\left\{v_{1} v_{2}\right\}-\left\{v_{1}\right\}-\left\{v_{2}\right\},\left(v_{1}, v_{2} \in F^{\times}\right)$. The quotient group $\mathcal{B}_{1}(F)=Z\left[\mathbf{P}_{F}^{1} /\{0,1, \infty\}\right] /\left\langle\mathrm{R}_{1}(F)\right\rangle$ is called classical polylogarithmic Bloch group for weight $n=1$. Bloch also defined a morphism $\delta_{1}: \mathcal{B}_{1}(F) \rightarrow F^{\times}$, defined as $\delta_{1}:[v] \rightarrow v$. This morphism is also an isomorphism. 
2.4.3. Polylog Bloch Group for Weight 2 and Bloch Suslin Chain Complex. Let $\mathrm{R}_{2}(F)=$ $\sum_{i=0}^{4}(-1)^{i} r\left(v_{0}, \ldots, \hat{v}_{i}, \ldots, v_{4}\right)$ is a subset of $Z\left[\mathbf{P}_{F}^{1} /\{0,1, \infty\}\right]$, generated by five term relation cross ratio of four points. This helped us to introduce weight 2 dilogarithm Bloch group $\mathcal{B}_{2}(F)=Z\left[\mathbf{P}_{F}^{1} /\{0,1, \infty\}\right] /\left\langle\mathrm{R}_{2}(F)\right\rangle$. We can now connect $\mathcal{B}_{2}(F)$ with $\wedge^{2} F^{\times}$to get the Bloch Suslin chain complex as follows [1].

$$
\mathcal{B}_{2}(F) \stackrel{\delta}{\longrightarrow} \wedge^{2} F^{\times}
$$

2.4.4. Goncharov Chain Complex for Weight-3. Goncharov [6-8,10] defined the following subgroup $\mathrm{R}_{3}(F)$, generated by the relation of triple cross ratio:

$$
\mathrm{R}_{3}(F)=\sum_{i=0}^{6}(-1)^{i} A l t_{6}\left[\frac{\left(v_{0}, v_{1}, v_{3}\right)\left(v_{1}, v_{2}, v_{4}\right)\left(v_{0}, v_{2}, v_{5}\right)}{\left(v_{0}, v_{1}, v_{4}\right)\left(v_{1}, v_{2}, v_{5}\right)\left(v_{0}, v_{2}, v_{3}\right)}\right] .
$$

Goncharov [7] defined Bloch group for weight 3 as

$$
\mathcal{B}_{3}(F)=Z\left[\mathbf{P}_{F}^{1} /\{0,1, \infty\}\right] /\left\langle\mathrm{R}_{3}(F)\right\rangle .
$$

Following is a chain complex for weight 3

$$
\mathbf{B}_{3}(F) \stackrel{\delta}{\longrightarrow} \mathcal{B}_{2}(F) \otimes F^{\times} \stackrel{\delta}{\longrightarrow} \wedge^{3} F^{\times}
$$

Lemma 2.5.

$$
\delta \circ \delta=0(\text { see }[7]) \text {. }
$$

2.5.1. Weight- $n$. Goncharov [7] extended his work and defined generalized Bloch group $\mathcal{B}_{n}(F)=Z\left[\mathbf{P}_{F}^{1} /\{0,1, \infty\}\right] /\left\langle\mathrm{R}_{n}(F)\right\rangle$, where $\mathrm{R}_{n}(F)$ is a subgroup of $Z\left[\mathbf{P}_{F}^{1}\right]$ and it is the kernel of the map $\delta_{n}: Z\left[\mathbf{P}_{F}^{1} /\{0,1, \infty\}\right] \rightarrow \mathcal{B}_{n-1}(F) \otimes F^{\times}$. Following is generalized polylogarithm chain complex called Goncharov chain complex.

$\mathcal{B}_{n}(F) \stackrel{\delta_{n}}{\longrightarrow} \mathcal{B}_{n-1}(F) \otimes F^{\times} \stackrel{\delta_{n-1}}{\longrightarrow} \mathcal{B}_{n-2}(F) \otimes \wedge^{2}(F) \stackrel{\delta_{n-2}}{\longrightarrow} \ldots \stackrel{\delta_{2}}{\longrightarrow} \mathcal{B}_{2}(F) \otimes \wedge^{n-2}(F) \stackrel{\delta_{1}}{\longrightarrow} \wedge^{n}\left(F^{\times}\right)$.

Lemma 2.6.

$$
\delta_{n-1} \circ \delta_{n}=0 \text { (see [7]). }
$$

\subsection{Generalized Tangent Group \& Tangential Chain Complex.}

2.7.1. Tangent Group of First Order. Let $x$ and $x^{\prime} \in F$ and $\left\langle x ; x^{\prime}\right]_{2}=\left[x+x^{\prime} \varepsilon\right]-[x] \in$ $\mathbb{Z}[F[\varepsilon]]_{2}[11,21]$. Initially, Cathelineau [4] introduced first order tangent group $T \mathcal{B}_{2}(F)$. This group is a quotient of Z-module generated by the elements $\left\langle x ; x^{\prime}\right]_{2} \in \mathbb{Z}[F[\varepsilon]]_{2}$ and the following five term relation

$$
\left\langle x ; x^{\prime}\right]-\left\langle y ; y^{\prime}\right]+\left\langle\frac{y}{x} ;\left(\frac{y}{x}\right)^{\prime}\right]-\left\langle\frac{1-y}{1-x} ;\left(\frac{1-y}{1-x}\right)^{\prime}\right]+\left\langle\frac{x(1-y)}{y(1-x)} ;\left(\frac{x(1-y)}{y(1-x)}\right)^{\prime}\right],
$$

$x \neq y$ and $x, y \neq 0,1[4,11]$. 
2.7.2. Tangential Chain Complex to Bloch Suslin Complex for Weight 2. In [4] for weight 2, Cathelineau defined the following tangential chain complex

$$
T \mathcal{B}_{2}(F) \stackrel{\delta_{\varepsilon}}{\longrightarrow} F \otimes F^{\times} \oplus \wedge^{2} F^{\times},
$$

where

$$
\delta_{\varepsilon}:\langle x, y]_{2}=\left(\frac{y}{x} \otimes(1-x)+\frac{y}{(1-x)} \otimes x\right)+\left(\frac{y}{(1-x)} \wedge \frac{y}{x}\right),
$$

see $[4,21]$.

2.7.3. Tangential Chain Complex to Goncharov chain complex for Weight 3. Cathelineau [4] also defined tangent group of order $3, T B_{3}(F)=\mathbb{Z}\left[F[\varepsilon]_{2}\right] /$ ker $_{3}$, ker $_{3}$ is the kernel of the map $\delta_{\varepsilon 3}=\mathbb{Z}\left[F[\varepsilon]_{2}\right] \rightarrow T B_{2}(F) \otimes F^{\times} \oplus F \otimes \mathcal{B}_{2}(F)$, then he defined the tangential chain complex to Goncharov complex for weight $n=3$ as

$$
T \mathcal{B}_{3}(F) \stackrel{\delta_{\varepsilon}}{\longrightarrow} T \mathcal{B}_{2}(F) \otimes F^{\times} \oplus F \otimes \mathcal{B}_{2}(F) \stackrel{\delta_{\varepsilon}}{\longrightarrow} F \otimes \wedge^{2} F^{\times} \oplus \wedge^{3} F^{\times} .
$$

2.7.4. Generalized Tangential Chain Complex. Cathelineau [4] generalized tangent group $T B_{n}(F)$ and then generalized the tangential chain complex to generalized Goncharov chain complex for any weight $n$ as

$T \mathcal{B}_{n}(F) \stackrel{\delta_{n, \varepsilon}}{\longrightarrow} \underset{F \otimes \mathcal{B}_{n-1}(F) \otimes F^{\times}}{\oplus} \stackrel{\delta_{(n-1), \varepsilon}}{\longrightarrow} \cdots \stackrel{\delta_{1, \varepsilon}}{\longrightarrow} \begin{gathered}T \mathcal{B}_{2}(F) \otimes \wedge^{\oplus-2} F^{\times} \\ F \otimes \mathcal{B}_{2}(F) \otimes \wedge^{n-3} F^{\times}\end{gathered} \stackrel{\delta_{\varepsilon}}{\longrightarrow}\left(F \otimes \wedge^{n-1} F^{\times}\right) \oplus\left(\wedge^{n} F\right)$ where $\delta_{(n-1), \varepsilon} \circ \delta_{n, \varepsilon}=0$ (see [4]).

\subsection{Tangential and Affine Configuration Geometry up to Weight 5.}

2.8.1. Weight 2 Geometry. Siddiqui in [21] defined the following geometry of affine configuration and tangential chain complexes for weight-2.

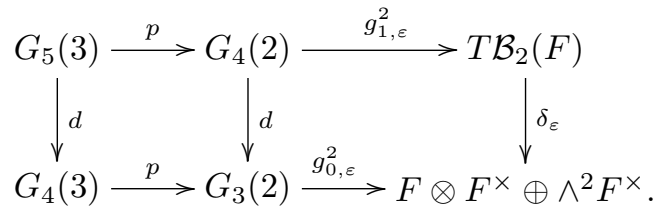

The spaces $G_{3}(2)$ and $G_{2}(2)$ used in diagram (C) are example of Euclidean space of dimension 3 and 2 .

Lemma 2.9.

$$
g_{0 \varepsilon}^{2} \circ d=\delta_{\varepsilon} \circ g_{1 \varepsilon}^{2}(\text { see }[21]) .
$$

2.9.1. Weight 3 Geometry. The geometry of for weight 3 is defined in [21] as follows:

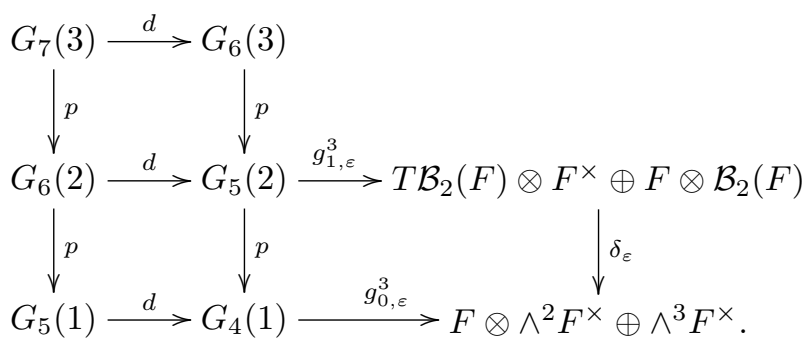


Lemma 2.10.

$g_{0 \varepsilon}^{3} \circ d=\delta_{\varepsilon} \circ g_{1 \varepsilon}^{3}($ see $[21])$.

2.10.1. Weight 4 Geometry. Khalid et al. [20] defined geometry for weight 4 as follows

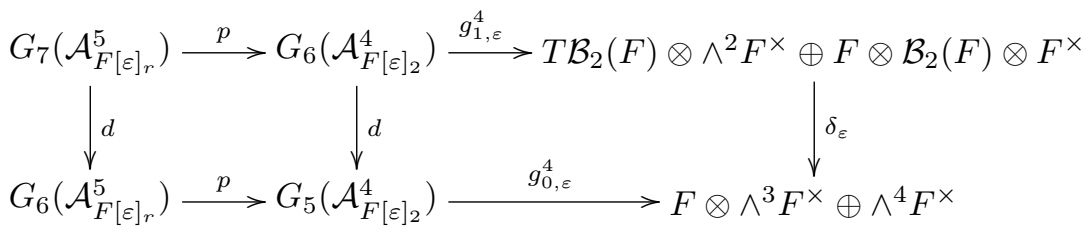

where, $g_{0 \varepsilon}^{4}\left(v_{0}^{*}, \ldots, v_{4}^{*}\right)=g_{0_{1}}^{4}\left(v_{0}^{*}, \ldots, v_{4}^{*}\right)+g_{0_{2}}^{4}\left(v_{0}^{*}, \ldots, v_{4}^{*}\right)$,

$$
\begin{array}{r}
g_{0_{1}}^{4}\left(v_{0}^{*}, \ldots, v_{4}^{*}\right)=\sum_{i=j+1}^{4}(-1)^{i+1} \frac{\triangle\left(v_{0}^{*}, \ldots, \hat{v}^{*}{ }_{i}, \ldots, v_{4}^{*}\right) \varepsilon}{\triangle\left(v_{0}, \ldots, \hat{v}_{i}, \ldots, v_{4}\right)} \otimes \frac{\triangle\left(v_{0}, \ldots, \hat{v}_{i+1}, \ldots, v_{4}\right)}{\triangle\left(v_{0}, \ldots, \hat{v}_{i+2}, \ldots, v_{4}\right)} \wedge \\
\frac{\triangle\left(v_{0}, \ldots, \hat{v}_{i+2}, \ldots, v_{4}\right)}{\triangle\left(v_{0}, \ldots, \hat{v}_{i+3}, \ldots, v_{4}\right)} \wedge \frac{\triangle\left(v_{0}, \ldots, \hat{v}_{i+3}, \ldots, v_{4}\right)}{\triangle\left(v_{0}, \ldots, \hat{v}_{i+4}, \ldots, v_{4}\right)}(i \bmod 5), \\
g_{0_{2}}^{4}\left(v_{0}^{*}, \ldots, v_{4}^{*}\right)= \\
\sum_{j=0}^{4}(-1)^{j+1} \bigwedge_{\substack{j \neq i \\
j=0}}^{4} \frac{\triangle\left(v_{0}^{*}, \ldots, \hat{v}^{*}{ }_{j}, \ldots, v_{4}^{*}\right) \varepsilon}{\triangle\left(v_{0}, \ldots, \hat{v}_{j}, \ldots, v_{4}\right)}(i \bmod 5)
\end{array}
$$

and

$$
\begin{aligned}
& g_{1 \varepsilon}^{4}\left(v_{0}^{*}, \ldots, v_{5}^{*}\right)=-\frac{1}{10} \sum_{i \neq j}^{5}(-1)^{i}\left(\left\langler\left(v_{i}, v_{j} \mid v_{0}, \ldots, \hat{v}_{i}, \hat{v}_{j}, \ldots, v_{5}\right)\right.\right. \\
& \left.r_{\varepsilon}\left(v_{i}^{*}, v_{j}^{*} \mid v_{0}^{*}, \ldots, \hat{v}^{*}{ }_{i}, \hat{v}^{*}{ }_{j}, \ldots, v_{5}^{*}\right)\right]_{2} \otimes \\
& \prod_{i \neq r}^{5} \triangle\left(v_{0}, \ldots, \hat{v}_{i}, \hat{v}_{r}, \ldots, v_{5}\right) \wedge \\
& \prod_{j \neq r}^{5} \triangle\left(v_{0}, \ldots, \hat{v}_{j}, \hat{v}_{r}, \ldots, v_{5}\right)+ \\
& \sum_{\substack{i \neq r \\
i=0}}^{5} \frac{\triangle\left(v_{0}^{*}, \ldots, \hat{v}^{*}{ }_{i}, \hat{v}^{*}{ }_{r}, \ldots, v_{5}^{*}\right)_{\varepsilon}}{\triangle\left(v_{0}, \ldots, \hat{v}_{i}, \hat{v}_{r}, \ldots, v_{5}\right)} \otimes \\
& {\left[r\left(v_{i}, v_{j} \mid v_{0}, \ldots, \hat{v}_{i}, \hat{v}_{j}, \ldots, v_{5}\right)\right]_{2} \otimes} \\
& \prod_{j \neq r}^{5} \triangle\left(v_{0}, \ldots, \hat{v}_{j}, \hat{v}_{r}, \ldots, v_{5}\right)+ \\
& \sum_{\substack{j \neq r \\
j=0}}^{5} \frac{\triangle\left(v_{0}^{*}, \ldots, \hat{v}^{*}{ }_{j}, \hat{v}^{*}{ }_{r}, \ldots, v_{5}^{*}\right)_{\varepsilon}}{\triangle\left(v_{0}, \ldots, \hat{v}_{j}, \hat{v}_{r}, \ldots, v_{5}\right)} \otimes \\
& {\left[r\left(v_{i}, v_{j} \mid v_{0}, \ldots, \hat{v}_{i}, \hat{v}_{j}, \ldots, v_{5}\right)\right]_{2} \otimes}
\end{aligned}
$$




$$
\left.\prod_{i \neq r}^{5} \triangle\left(v_{0}, \ldots, \hat{v}_{i}, \hat{v}_{r}, \ldots, v_{5}\right)\right)(\bmod 6) .
$$

Lemma 2.11. The above diagram $\mathrm{E}$ is commutative (see [20].)

2.11.1. Weight 5 Geometry. Khalid et al. [20] extended their work and defined geometry for weight 5 as follows

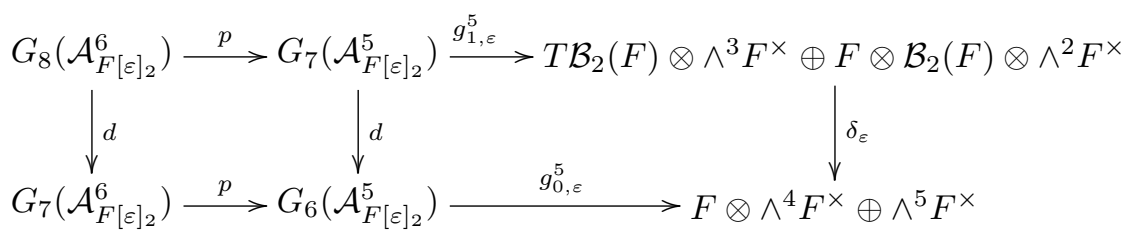

where, $g_{0 \varepsilon}^{5}\left(v_{0}^{*}, \ldots, v_{5}^{*}\right)=g_{0_{1}}^{5}\left(v_{0}^{*}, \ldots, v_{5}^{*}\right)+g_{0_{2}}^{5}\left(v_{0}^{*}, \ldots, v_{5}^{*}\right)$

$$
\begin{aligned}
& g_{0_{1}}^{5}\left(v_{0}^{*}, \ldots, v_{5}^{*}\right)=\sum_{i=j+1}^{5}(-1)^{i+1} \frac{\triangle\left(v_{0}^{*}, \ldots, \hat{v}^{*}{ }_{i}, \ldots, v_{5}^{*}\right) \varepsilon}{\triangle\left(v_{0}, \ldots, \hat{v}_{i}, \ldots, v_{5}\right)} \otimes \frac{\triangle\left(v_{0}, \ldots, \hat{v}_{i+1}, \ldots, v_{5}\right)}{\triangle\left(v_{0}, \ldots, \hat{v}_{i+2}, \ldots, v_{5}\right)} \wedge \\
& \frac{\triangle\left(v_{0}, \ldots, \hat{v}_{i+2}, \ldots, v_{5}\right)}{\triangle\left(v_{0}, \ldots, \hat{v}_{i+3}, \ldots, v_{5}\right)} \wedge \frac{\triangle\left(v_{0}, \ldots, \hat{v}_{i+3}, \ldots, v_{5}\right)}{\triangle\left(v_{0}, \ldots, \hat{v}_{i+4}, \ldots, v_{5}\right)} \wedge \\
& \frac{\triangle\left(v_{0}, \ldots, \hat{v}_{i+4}, \ldots, v_{5}\right)}{\triangle\left(v_{0}, \ldots, \hat{v}_{i+5}, \ldots, v_{5}\right)}(i \bmod 6), \\
& g_{0_{2}}^{5}\left(v_{0}^{*}, \ldots, v_{5}^{*}\right)=\sum_{j=0}^{5}(-1)^{j+1} \bigwedge_{\substack{j \neq i \\
j=0}}^{5} \frac{\triangle\left(v_{0}^{*}, \ldots, \hat{v}^{*}{ }_{j}, \ldots, v_{5}^{*}\right) \varepsilon}{\triangle\left(v_{0}, \ldots, \hat{v}_{j}, \ldots, v_{5}\right)}(i \bmod 6)
\end{aligned}
$$

and

$$
\begin{gathered}
g_{1 \varepsilon}^{5}\left(v_{0}^{*}, \ldots, v_{6}^{*}\right)=\frac{1}{15} \sum_{i \neq j}^{6}(-1)^{i}\left(\left\langler\left(v_{i}, v_{j}, v_{j} \mid v_{0}, \ldots, \hat{v}_{i}, \hat{v}_{j}, \hat{v}_{k}, \ldots, v_{6}\right) ;\right.\right. \\
\left.r_{\varepsilon}\left(v_{i}^{*}, v_{j}^{*}, v_{k}^{*} \mid v_{0}^{*}, \ldots, \hat{v}^{*}{ }_{i}, \hat{v}^{*}{ }_{j}, \hat{v}^{*}{ }_{k}, \ldots, v_{6}^{*}\right)\right]_{2} \otimes \\
\prod_{i \neq r \neq s}^{6} \triangle\left(v_{0}, \ldots, \hat{v}_{i}, \hat{v}_{r}, \hat{v}_{s}, \ldots, v_{6}\right) \wedge \\
\prod_{j \neq r \neq s}^{6} \triangle\left(v_{0}, \ldots, \hat{v}_{j}, \hat{v}_{r}, \hat{v}_{s}, \ldots, v_{6}\right) \wedge \\
\prod_{k \neq r \neq s}^{6} \triangle\left(v_{0}, \ldots, \hat{v}_{k}, \hat{v}_{r}, \hat{v}_{s}, \ldots, v_{6}\right)+ \\
\sum_{\substack{i \neq r \neq s \\
i=0}}^{6} \frac{\triangle\left(v_{0}^{*}, \ldots, \hat{v}^{*} i, \hat{v}^{*} r\right.}{\triangle\left(v_{0}, \ldots, \hat{v}_{i}{ }^{*}, \hat{v}_{r}, \ldots, \hat{v}_{s}, \ldots, v_{6}\right) \varepsilon} \otimes
\end{gathered}
$$




$$
\begin{aligned}
& {\left[r\left(v_{i}, v_{j}, v_{k} \mid v_{0}, \ldots, \hat{v}_{i}, \hat{v}_{j}, \hat{v}_{k}, \ldots, v_{6}\right)\right]_{2} \otimes} \\
& \prod_{j \neq r \neq s}^{6} \triangle\left(v_{0}, \ldots, \hat{v}_{j}, \hat{v}_{r}, \hat{v}_{s}, \ldots, v_{6}\right) \wedge \\
& \prod_{k \neq r \neq s}^{6} \triangle\left(v_{0}, \ldots, \hat{v}_{k}, \hat{v}_{r}, \hat{v}_{s}, \ldots, v_{6}\right)+ \\
& \sum_{\substack{j \neq r \neq s \\
j=0}}^{6} \frac{\triangle\left(v_{0}^{*}, \ldots, \hat{v}^{*}{ }_{j}, \hat{v}^{*}{ }_{r}, \hat{v}^{*}{ }_{s}, \ldots, v_{6}^{*}\right) \varepsilon}{\triangle\left(v_{0}, \ldots, \hat{v}_{j}, \hat{v}_{r}, \hat{v}_{s}, \ldots, v_{6}\right)} \otimes \\
& {\left[r\left(v_{i}, v_{j}, v_{k} \mid v_{0}, \ldots, \hat{v}_{i}, \hat{v}_{j}, \hat{v}_{k}, \ldots, v_{6}\right)\right]_{2} \otimes} \\
& \prod_{k \neq r \neq s}^{6} \triangle\left(v_{0}, \ldots, \hat{v}_{k}, \hat{v}_{r}, \hat{v}_{s}, \ldots, v_{6}\right) \wedge \\
& \prod_{i \neq r \neq s}^{6} \triangle\left(v_{0}, \ldots, \hat{v}_{i}, \hat{v}_{r}, \hat{v}_{s}, \ldots, v_{6}\right)+ \\
& \sum_{\substack{k \neq r \neq s \\
k=0}}^{6} \frac{\triangle\left(v_{0}^{*}, \ldots, \hat{v}^{*}{ }_{k}, \hat{v}^{*}{ }_{r}, \hat{v}^{*}{ }_{s}, \ldots, v_{6}^{*}\right) \varepsilon}{\triangle\left(v_{0}, \ldots, \hat{v}_{k}, \hat{v}_{r}, \hat{v}_{s}, \ldots, v_{6}\right)} \otimes \\
& {\left[r\left(v_{i}, v_{j}, v_{k} \mid v_{0}, \ldots, \hat{v}_{i}, \hat{v}_{j}, \hat{v}_{k}, \ldots, v_{6}\right)\right]_{2} \otimes} \\
& \prod_{i \neq r \neq s}^{6} \triangle\left(v_{0}, \ldots, \hat{v}_{i}, \hat{v}_{r}, \hat{v}_{s}, \ldots, v_{6}\right) \wedge \\
& \prod_{j \neq r \neq s}^{6} \triangle\left(v_{0}, \ldots, \hat{v}_{j}, \hat{v}_{r}, \hat{v}_{s}, \ldots, v_{6}\right)(\bmod 6) .
\end{aligned}
$$

Lemma 2.12. The right square of diagram $\mathrm{F}$ is commutative (see [20].)

\section{Geometry for Weight $n=6 \&$ Generalization}

3.1. Geometry for Weight 6. For weight 6 , we construct the following commutative diagram

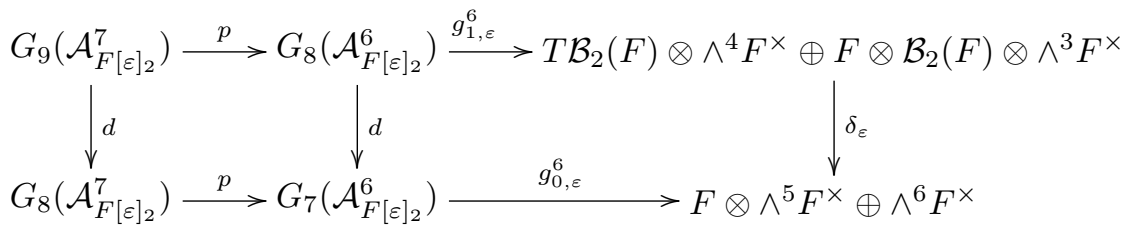

where, $g_{0 \varepsilon}^{6}\left(v_{0}^{*}, \ldots, v_{6}^{*}\right)=g_{0_{1}}^{6}\left(v_{0}^{*}, \ldots, v_{6}^{*}\right)+g_{0_{2}}^{6}\left(v_{0}^{*}, \ldots, v_{6}^{*}\right)$,

$g_{0_{1}}^{6}\left(v_{0}^{*}, \ldots, v_{6}^{*}\right)=\sum_{i=j+1}^{6}(-1)^{i+1} \frac{\triangle\left(v_{0}^{*}, \ldots, \hat{v}^{*}{ }_{i}, \ldots, v_{6}^{*}\right) \varepsilon}{\triangle\left(v_{0}, \ldots, \hat{v}_{i}, \ldots, v_{6}\right)} \otimes \frac{\triangle\left(v_{0}, \ldots, \hat{v}_{i+1}, \ldots, v_{6}\right)}{\triangle\left(v_{0}, \ldots, \hat{v}_{i+2}, \ldots, v_{6}\right)} \wedge$ 


$$
\begin{aligned}
& \frac{\triangle\left(v_{0}, \ldots, \hat{v}_{i+2}, \ldots, v_{6}\right)}{\triangle\left(v_{0}, \ldots, \hat{v}_{i+3}, \ldots, v_{6}\right)} \wedge \frac{\triangle\left(v_{0}, \ldots, \hat{v}_{i+3}, \ldots, v_{6}\right)}{\triangle\left(v_{0}, \ldots, \hat{v}_{i+4}, \ldots, v_{6}\right)} \wedge \\
& \frac{\triangle\left(v_{0}, \ldots, \hat{v}_{i+4}, \ldots, v_{6}\right)}{\triangle\left(v_{0}, \ldots, \hat{v}_{i+5}, \ldots, v_{6}\right)} \wedge \frac{\triangle\left(v_{0}, \ldots, \hat{v}_{i+5}, \ldots, v_{6}\right)}{\triangle\left(v_{0}, \ldots, \hat{v}_{i+6}, \ldots, v_{6}\right)}(i \bmod 7)
\end{aligned}
$$

$$
g_{0_{2}}^{6}\left(v_{0}^{*}, \ldots, v_{6}^{*}\right)=\sum_{j=0}^{6}(-1)^{j+1} \bigwedge_{\substack{j \neq i \\ j=0}}^{6} \frac{\triangle\left(v_{0}^{*}, \ldots, \hat{v}^{*}{ }_{j}, \ldots, v_{6}^{*}\right) \varepsilon}{\triangle\left(v_{0}, \ldots, \hat{v}_{j}, \ldots, v_{6}\right)}(i \bmod 7)
$$

and

$$
\begin{aligned}
& \begin{array}{r}
g_{1 \varepsilon}^{6}\left(v_{0}^{*}, \ldots, v_{7}^{*}\right)=-\frac{1}{21} \sum_{i \neq j}^{7}(-1)^{i}\left(\left\langler \left(v_{i}, v_{j}, v_{k}, v_{l} \mid v_{0}, \ldots, \hat{v}_{i}, \hat{v}_{j}, \hat{v}_{k}, \hat{v}_{k}, \hat{v}_{l}, \ldots,\right.\right.\right. \\
\left.\left.v_{k}^{*}, v_{l}^{*} \mid v_{0}^{*}, \ldots, \hat{v}^{*}{ }_{i}, \hat{v}^{*}{ }_{j}, \hat{v}^{*}{ }_{k}, \hat{v}^{*}{ }_{l}, \ldots, v_{7}^{*}\right)\right]_{2} \otimes
\end{array} \\
& \prod_{i \neq r \neq s \neq t}^{7} \triangle\left(v_{0}, \ldots, \hat{v}_{i}, \hat{v}_{r}, \hat{v}_{s}, \hat{v}_{t}, \ldots, v_{7}\right) \wedge \\
& \prod_{j \neq r \neq s \neq t}^{7} \triangle\left(v_{0}, \ldots, \hat{v}_{j}, \hat{v}_{r}, \hat{v}_{s}, \hat{v}_{t}, \ldots, v_{7}\right) \wedge \\
& \prod_{k \neq r \neq s \neq t}^{7} \triangle\left(v_{0}, \ldots, \hat{v}_{k}, \hat{v}_{r}, \hat{v}_{s}, \hat{v}_{t}, \ldots, v_{7}\right) \wedge \\
& \prod_{l \neq r \neq s \neq t}^{7} \triangle\left(v_{0}, \ldots, \hat{v}_{l}, \hat{v}_{r}, \hat{v}_{s}, \hat{v}_{t}, \ldots, v_{7}\right)+ \\
& \sum_{\substack{i \neq r \neq s \neq t \\
i=0}}^{7} \frac{\triangle\left(v_{0}^{*}, \ldots, \hat{v}^{*}{ }_{i}, \hat{v}^{*}{ }_{r}, \hat{v}^{*}{ }_{s}, \hat{v}^{*}{ }_{t}, \ldots, v_{7}^{*}\right) \varepsilon}{\triangle\left(v_{0}, \ldots, \hat{v}_{i}, \hat{v}_{r}, \hat{v}_{s}, \hat{v}_{t}, \ldots, v_{7}\right)} \otimes \\
& {\left[r\left(v_{i}, v_{j}, v_{k}, v_{l} \mid v_{0}, \ldots, \hat{v}_{i}, \hat{v}_{j}, \hat{v}_{k}, \hat{v}_{l}, \ldots, v_{7}\right)\right]_{2} \otimes} \\
& \prod_{j \neq r \neq s \neq t}^{7} \triangle\left(v_{0}, \ldots, \hat{v}_{j}, \hat{v}_{r}, \hat{v}_{s}, \hat{v}_{t}, \ldots, v_{7}\right) \wedge \\
& \prod_{k \neq r \neq s \neq t}^{7} \triangle\left(v_{0}, \ldots, \hat{v}_{k}, \hat{v}_{r}, \hat{v}_{s}, \hat{v}_{t}, \ldots, v_{7}\right) \wedge \\
& \prod_{l \neq r \neq s \neq t}^{7} \triangle\left(v_{0}, \ldots, \hat{v}_{l}, \hat{v}_{r}, \hat{v}_{s}, \hat{v}_{t}, \ldots, v_{7}\right)+ \\
& \sum_{\substack{j \neq r \neq s \neq t \\
j=0}}^{7} \frac{\triangle\left(v_{0}^{*}, \ldots, \hat{v}^{*}{ }_{j}, \hat{v}^{*}{ }_{r}, \hat{v}^{*}{ }_{s}, \hat{v}^{*}{ }_{t}, \ldots, v_{7}^{*}\right) \varepsilon}{\triangle\left(v_{0}, \ldots, \hat{v}_{j}, \hat{v}_{r}, \hat{v}_{s}, \hat{v}_{t}, \ldots, v_{7}\right)} \otimes
\end{aligned}
$$




$$
\begin{aligned}
& {\left[r\left(v_{i}, v_{j}, v_{k}, v_{l} \mid v_{0}, \ldots, \hat{v}_{i}, \hat{v}_{j}, \hat{v}_{k}, \hat{v}_{l}, \ldots, v_{7}\right)\right]_{2} \otimes} \\
& \prod_{i \neq r \neq s \neq t}^{7} \triangle\left(v_{0}, \ldots, \hat{v}_{i}, \hat{v}_{r}, \hat{v}_{s}, \hat{v}_{t}, \ldots, v_{7}\right) \wedge \\
& \prod_{k \neq r \neq s \neq t}^{7} \triangle\left(v_{0}, \ldots, \hat{v}_{k}, \hat{v}_{r}, \hat{v}_{s}, \hat{v}_{t}, \ldots, v_{7}\right) \wedge \\
& \prod_{l \neq r \neq s \neq t}^{7} \triangle\left(v_{0}, \ldots, \hat{v}_{l}, \hat{v}_{r}, \hat{v}_{s}, \hat{v}_{t}, \ldots, v_{7}\right)+ \\
& \sum_{\substack{k \neq r \neq s \neq t \\
k=0}}^{7} \frac{\triangle\left(v_{0}^{*}, \ldots, \hat{v}^{*}{ }_{k}, \hat{v}^{*}{ }_{r}, \hat{v}^{*}{ }_{s}, \hat{v}^{*}{ }_{t}, \ldots, v_{7}^{*}\right) \varepsilon}{\triangle\left(v_{0}, \ldots, \hat{v}_{k}, \hat{v}_{r}, \hat{v}_{s}, \hat{v}_{t}, \ldots, v_{7}\right)} \otimes \\
& {\left[r\left(v_{i}, v_{j}, v_{k}, v_{l} \mid v_{0}, \ldots, \hat{v}_{i}, \hat{v}_{j}, \hat{v}_{k}, \hat{v}_{l}, \ldots, v_{7}\right)\right]_{2} \otimes} \\
& \prod_{i \neq r \neq s \neq t}^{7} \triangle\left(v_{0}, \ldots, \hat{v}_{i}, \hat{v}_{r}, \hat{v}_{s}, \hat{v}_{t}, \ldots, v_{7}\right) \wedge \\
& \prod_{j \neq r \neq s \neq t}^{7} \triangle\left(v_{0}, \ldots, \hat{v}_{j}, \hat{v}_{r}, \hat{v}_{s}, \hat{v}_{t}, \ldots, v_{7}\right) \wedge \\
& \prod_{l \neq r \neq s \neq t}^{7} \triangle\left(v_{0}, \ldots, \hat{v}_{l}, \hat{v}_{r}, \hat{v}_{s}, \hat{v}_{t}, \ldots, v_{7}\right)+ \\
& \sum_{\substack{l \neq r \neq s \neq t \\
l=0}}^{7} \frac{\triangle\left(v_{0}^{*}, \ldots, \hat{v}^{*} l, \hat{v}^{*} r, \hat{v}^{*}{ }_{s}, \hat{v}^{*}{ }_{t}, \ldots, v_{7}^{*}\right) \varepsilon}{\triangle\left(v_{0}, \ldots, \hat{v}_{l}, \hat{v}_{r}, \hat{v}_{s}, \hat{v}_{t}, \ldots, v_{7}\right)} \otimes \\
& {\left[r\left(v_{i}, v_{j}, v_{k}, v_{l} \mid v_{0}, \ldots, \hat{v}_{i}, \hat{v}_{j}, \hat{v}_{k}, \hat{v}_{l}, \ldots, v_{7}\right)\right]_{2} \otimes} \\
& \prod_{i \neq r \neq s \neq t}^{7} \triangle\left(v_{0}, \ldots, \hat{v}_{i}, \hat{v}_{r}, \hat{v}_{s}, \hat{v}_{t}, \ldots, v_{7}\right) \wedge \\
& \prod_{j \neq r \neq s \neq t}^{7} \triangle\left(v_{0}, \ldots, \hat{v}_{j}, \hat{v}_{r}, \hat{v}_{s}, \hat{v}_{t}, \ldots, v_{7}\right) \wedge \\
& \prod_{k \neq r \neq s \neq t}^{7} \triangle\left(v_{0}, \ldots, \hat{v}_{k}, \hat{v}_{r}, \hat{v}_{s}, \hat{v}_{t}, \ldots, v_{7}\right)(\bmod 8) .
\end{aligned}
$$

Theorem 3.2. $\quad g_{0 \varepsilon}^{6} \circ d=\delta_{\varepsilon} \circ g_{1 \varepsilon}^{6}$ 
Proof. Proof of the above theorem is same as lemma (3.2) and lemma (3.4) of [20]. Let $\left(v_{0}^{*}, \ldots, v_{7}^{*}\right) \in G_{8}\left(\mathcal{A}_{F[\varepsilon]_{2}}^{6}\right)$ and apply morphism $d$, then we get

$$
d\left(v_{0}^{*}, \ldots, v_{7}^{*}\right)=\sum_{i=0}^{7}(-1)^{i}\left(v_{0}^{*}, \ldots, \hat{v}_{i}, \ldots, v_{7}^{*}\right)
$$

Now compose morphism $g_{0_{1}}^{6}$ with $d\left(v_{0}^{*}, \ldots, v_{7}^{*}\right)$ then

$$
\begin{aligned}
g_{0_{1}}^{6} \circ d\left(v_{0}^{*}, \ldots, v_{7}^{*}\right)= & \sum_{j=i+1}^{7-i}(-1)^{j} \sum_{i=0}^{7}(-1)^{i} \frac{\triangle\left(v_{0}^{*}, \ldots, \hat{v}^{*}{ }_{j}, \hat{v}^{*} i, \ldots, v_{7}^{*}\right) \varepsilon}{\triangle\left(v_{0}, \ldots, \hat{v}_{j}, \hat{v}_{i}, \ldots, v_{7}\right)} \otimes \\
& \frac{\triangle\left(v_{0}, \ldots, \hat{v}_{j+1}, \hat{v}_{i}, \ldots, v_{7}\right)}{\triangle\left(v_{0}, \ldots, \hat{v}_{j+2}, \hat{v}_{i}, \ldots, v_{7}\right)} \wedge \frac{\triangle\left(v_{0}, \ldots, \hat{v}_{j+2}, \hat{v}_{i}, \ldots, v_{7}\right)}{\triangle\left(v_{0}, \ldots, \hat{v}_{j+3}, \hat{v}_{i}, \ldots, v_{7}\right)} \wedge \\
& \frac{\triangle\left(v_{0}, \ldots, \hat{v}_{j+3}, \hat{v}_{i}, \ldots, v_{7}\right)}{\triangle\left(v_{0}, \ldots, \hat{v}_{j+4}, \hat{v}_{i}, \ldots, v_{7}\right)} \wedge \frac{\triangle\left(v_{0}, \ldots, \hat{v}_{j+4}, \hat{v}_{i}, \ldots, v_{7}\right)}{\triangle\left(v_{0}, \ldots, \hat{v}_{j+5}, \hat{v}_{i}, \ldots, v_{7}\right)} \wedge \\
& \frac{\triangle\left(v_{0}, \ldots, \hat{v}_{j+5}, \hat{v}_{i}, \ldots, v_{7}\right)}{\triangle\left(v_{0}, \ldots, \hat{v}_{j+6}, \hat{v}_{i}, \ldots, v_{7}\right)},
\end{aligned}
$$

now apply morphism $g_{0_{2}}^{6}$ then

$$
g_{0_{2}}^{6} \circ d\left(v_{0}^{*}, \ldots, v_{7}^{*}\right)=\sum_{j=0}^{7-i}(-1)^{j+1} \sum_{i=0}^{7}(-1)^{i} \bigwedge_{\substack{j \neq i \\ j=0}}^{7} \frac{\triangle\left(v_{0}^{*}, \ldots, \hat{v}^{*}, \hat{v}^{*}{ }_{i}, \ldots, v_{7}^{*}\right) \varepsilon}{\triangle\left(v_{0}, \ldots, \hat{v}_{j}, \hat{v}_{i}, \ldots, v_{7}\right) .}
$$

As morphism $g_{0 \varepsilon}^{6}=g_{0_{1}}^{6}+g_{0_{2}}^{6}$, therefore combine Eq.(3.18) and Eq.(3.19), we get

$$
\begin{aligned}
g_{0 \varepsilon}^{6} \circ d\left(v_{0}^{*}, \ldots, v_{7}^{*}\right)= & \sum_{j=i+1}^{7-i}(-1)^{j} \sum_{i=0}^{7}(-1)^{i} \frac{\triangle\left(v_{0}^{*}, \ldots, \hat{v}^{*}{ }_{j}, \hat{v}^{*} i, \ldots, v_{7}^{*}\right) \varepsilon}{\triangle\left(v_{0}, \ldots, \hat{v}_{j}, \hat{v}_{i}, \ldots, v_{7}\right)} \otimes \\
& \frac{\triangle\left(v_{0}, \ldots, \hat{v}_{j+1}, \hat{v}_{i}, \ldots, v_{7}\right)}{\triangle\left(v_{0}, \ldots, \hat{v}_{j+2}, \hat{v}_{i}, \ldots, v_{7}\right)} \wedge \frac{\triangle\left(v_{0}, \ldots, \hat{v}_{j+2}, \hat{v}_{i}, \ldots, v_{7}\right)}{\triangle\left(v_{0}, \ldots, \hat{v}_{j+3}, \hat{v}_{i}, \ldots, v_{7}\right)} \wedge \\
& \frac{\triangle\left(v_{0}, \ldots, \hat{v}_{j+3}, \hat{v}_{i}, \ldots, v_{7}\right)}{\triangle\left(v_{0}, \ldots, \hat{v}_{j+4}, \hat{v}_{i}, \ldots, v_{7}\right)} \wedge \frac{\triangle\left(v_{0}, \ldots, \hat{v}_{j+4}, \hat{v}_{i}, \ldots, v_{7}\right)}{\triangle\left(v_{0}, \ldots, \hat{v}_{j+5}, \hat{v}_{i}, \ldots, v_{7}\right)} \wedge \\
& \frac{\triangle\left(v_{0}, \ldots, \hat{v}_{j+5}, \hat{v}_{i}, \ldots, v_{7}\right)}{\triangle\left(v_{0}, \ldots, \hat{v}_{j+6}, \hat{v}_{i}, \ldots, v_{7}\right)}+ \\
& \sum_{j=0}^{7-i}(-1)^{j+1} \sum_{i=0}^{7}(-1)^{i} \bigwedge_{\substack{j \neq i \\
j=0}}^{7} \frac{\triangle\left(v_{0}^{*}, \ldots, \hat{v}^{*}{ }_{j}, \hat{v}^{*}{ }_{i}, \ldots, v_{7}^{*}\right) \varepsilon}{\triangle\left(v_{0}, \ldots, \hat{v}_{j}, \hat{v}_{i}, \ldots, v_{7}\right) .}
\end{aligned}
$$

Take again $\left(v_{0}^{*}, \ldots, v_{7}^{*}\right) \in G_{8}\left(\mathcal{A}_{F[\varepsilon]_{2}}^{6}\right)$ and apply morphism $g_{1 \varepsilon}^{6}$, we get

$$
\begin{array}{r}
g_{1 \varepsilon}^{6}\left(v_{0}^{*}, \ldots, v_{7}^{*}\right)=-\frac{1}{21} \sum_{i \neq j}^{7}(-1)^{i}\left(\left\langler\left(v_{i}, v_{j}, v_{k}, v_{l} \mid v_{0}, \ldots, \hat{v}_{i}, \hat{v}_{j}, \hat{v}_{k}, \hat{v}_{k}, \hat{v}_{l}, \ldots, v_{7}\right) ;\right.\right. \\
\left.r_{\varepsilon}\left(v_{i}^{*}, v_{j}^{*}, v_{k}^{*}, v_{l}^{*} \mid v_{0}^{*}, \ldots, \hat{v}^{*}, \hat{v}^{*}{ }_{j}, \hat{v}^{*} k, \hat{v}^{*} l, \ldots, v_{7}^{*}\right)\right]_{2} \otimes
\end{array}
$$




$$
\begin{aligned}
& \prod_{i \neq r \neq s \neq t}^{7} \triangle\left(v_{0}, \ldots, \hat{v}_{i}, \hat{v}_{r}, \hat{v}_{s}, \hat{v}_{t}, \ldots, v_{7}\right) \wedge \\
& \prod_{j \neq r \neq s \neq t}^{7} \triangle\left(v_{0}, \ldots, \hat{v}_{j}, \hat{v}_{r}, \hat{v}_{s}, \hat{v}_{t}, \ldots, v_{7}\right) \wedge \\
& \prod_{k \neq r \neq s \neq t}^{7} \triangle\left(v_{0}, \ldots, \hat{v}_{k}, \hat{v}_{r}, \hat{v}_{s}, \hat{v}_{t}, \ldots, v_{7}\right) \wedge \\
& \prod_{l \neq r \neq s \neq t}^{7} \triangle\left(v_{0}, \ldots, \hat{v}_{l}, \hat{v}_{r}, \hat{v}_{s}, \hat{v}_{t}, \ldots, v_{7}\right)+ \\
& \sum_{\substack{i \neq r \neq s \neq t \\
i=0}}^{7} \frac{\triangle\left(v_{0}^{*}, \ldots, \hat{v}^{*}{ }_{i}, \hat{v}^{*}{ }_{r}, \hat{v}^{*}{ }_{s}, \hat{v}^{*}{ }_{t}, \ldots, v_{7}^{*}\right) \varepsilon}{\triangle\left(v_{0}, \ldots, \hat{v}_{i}, \hat{v}_{r}, \hat{v}_{s}, \hat{v}_{t}, \ldots, v_{7}\right)} \otimes \\
& {\left[r\left(v_{i}, v_{j}, v_{k}, v_{l} \mid v_{0}, \ldots, \hat{v}_{i}, \hat{v}_{j}, \hat{v}_{k}, \hat{v}_{l}, \ldots, v_{7}\right)\right]_{2} \otimes} \\
& \prod_{j \neq r \neq s \neq t}^{7} \triangle\left(v_{0}, \ldots, \hat{v}_{j}, \hat{v}_{r}, \hat{v}_{s}, \hat{v}_{t}, \ldots, v_{7}\right) \wedge \\
& \prod_{k \neq r \neq s \neq t}^{7} \triangle\left(v_{0}, \ldots, \hat{v}_{k}, \hat{v}_{r}, \hat{v}_{s}, \hat{v}_{t}, \ldots, v_{7}\right) \wedge \\
& \prod_{l \neq r \neq s \neq t}^{7} \triangle\left(v_{0}, \ldots, \hat{v}_{l}, \hat{v}_{r}, \hat{v}_{s}, \hat{v}_{t}, \ldots, v_{7}\right)+ \\
& \sum_{\substack{j \neq r \neq s \neq t \\
j=0}}^{7} \frac{\triangle\left(v_{0}^{*}, \ldots, \hat{v}^{*}{ }_{j}, \hat{v}^{*}{ }_{r}, \hat{v}^{*}{ }_{s}, \hat{v}^{*}{ }_{t}, \ldots, v_{7}^{*}\right) \varepsilon}{\triangle\left(v_{0}, \ldots, \hat{v}_{j}, \hat{v}_{r}, \hat{v}_{s}, \hat{v}_{t}, \ldots, v_{7}\right)} \otimes \\
& {\left[r\left(v_{i}, v_{j}, v_{k}, v_{l} \mid v_{0}, \ldots, \hat{v}_{i}, \hat{v}_{j}, \hat{v}_{k}, \hat{v}_{l}, \ldots, v_{7}\right)\right]_{2} \otimes} \\
& \prod_{i \neq r \neq s \neq t}^{7} \triangle\left(v_{0}, \ldots, \hat{v}_{i}, \hat{v}_{r}, \hat{v}_{s}, \hat{v}_{t}, \ldots, v_{7}\right) \wedge \\
& \prod_{k \neq r \neq s \neq t}^{7} \triangle\left(v_{0}, \ldots, \hat{v}_{k}, \hat{v}_{r}, \hat{v}_{s}, \hat{v}_{t}, \ldots, v_{7}\right) \wedge \\
& \prod_{l \neq r \neq s \neq t}^{7} \triangle\left(v_{0}, \ldots, \hat{v}_{l}, \hat{v}_{r}, \hat{v}_{s}, \hat{v}_{t}, \ldots, v_{7}\right)+ \\
& \sum_{\substack{k \neq r \neq s \neq t \\
k=0}}^{7} \frac{\triangle\left(v_{0}^{*}, \ldots, \hat{v}^{*}{ }_{k}, \hat{v}^{*}{ }_{r}, \hat{v}^{*}{ }_{s}, \hat{v}^{*}{ }_{t}, \ldots, v_{7}^{*}\right) \varepsilon}{\triangle\left(v_{0}, \ldots, \hat{v}_{k}, \hat{v}_{r}, \hat{v}_{s}, \hat{v}_{t}, \ldots, v_{7}\right)} \otimes \\
& {\left[r\left(v_{i}, v_{j}, v_{k}, v_{l} \mid v_{0}, \ldots, \hat{v}_{i}, \hat{v}_{j}, \hat{v}_{k}, \hat{v}_{l}, \ldots, v_{7}\right)\right]_{2} \otimes}
\end{aligned}
$$




$$
\begin{aligned}
& \prod_{i \neq r \neq s \neq t}^{7} \triangle\left(v_{0}, \ldots, \hat{v}_{i}, \hat{v}_{r}, \hat{v}_{s}, \hat{v}_{t}, \ldots, v_{7}\right) \wedge \\
& \prod_{j \neq r \neq s \neq t}^{7} \triangle\left(v_{0}, \ldots, \hat{v}_{j}, \hat{v}_{r}, \hat{v}_{s}, \hat{v}_{t}, \ldots, v_{7}\right) \wedge \\
& \prod_{l \neq r \neq s \neq t}^{7} \triangle\left(v_{0}, \ldots, \hat{v}_{l}, \hat{v}_{r}, \hat{v}_{s}, \hat{v}_{t}, \ldots, v_{7}\right)+
\end{aligned}
$$

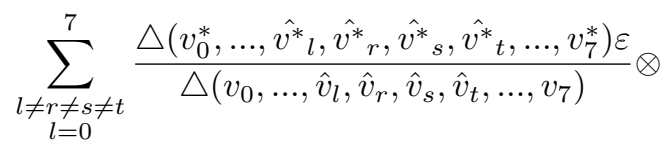

$$
\begin{aligned}
& {\left[r\left(v_{i}, v_{j}, v_{k}, v_{l} \mid v_{0}, \ldots, \hat{v}_{i}, \hat{v}_{j}, \hat{v}_{k}, \hat{v}_{l}, \ldots, v_{7}\right)\right]_{2} \otimes} \\
& \prod_{i \neq r \neq s \neq t}^{7} \triangle\left(v_{0}, \ldots, \hat{v}_{i}, \hat{v}_{r}, \hat{v}_{s}, \hat{v}_{t}, \ldots, v_{7}\right) \wedge \\
& \prod_{j \neq r \neq s \neq t}^{7} \triangle\left(v_{0}, \ldots, \hat{v}_{j}, \hat{v}_{r}, \hat{v}_{s}, \hat{v}_{t}, \ldots, v_{7}\right) \wedge \\
& \prod_{k \neq r \neq s \neq t}^{7} \triangle\left(v_{0}, \ldots, \hat{v}_{k}, \hat{v}_{r}, \hat{v}_{s}, \hat{v}_{t}, \ldots, v_{7}\right) .
\end{aligned}
$$

Now compose morphism $\delta_{\varepsilon}$ with $g_{1 \varepsilon}^{6}\left(v_{0}^{*}, \ldots, v_{7}^{*}\right)$ and simplify using tensor, cycle, Siegel cross ratio and wedge properties, the above becomes

$$
\begin{aligned}
\delta_{\varepsilon} \circ g_{1 \varepsilon}^{6}\left(v_{0}^{*}, \ldots, v_{7}^{*}\right)= & \sum_{j=i+1}^{7-i}(-1)^{j} \sum_{i=0}^{7}(-1)^{i} \frac{\triangle\left(v_{0}^{*}, \ldots, \hat{v}^{*}{ }_{j}, \hat{v}^{*}, \ldots, v_{7}^{*}\right) \varepsilon}{\triangle\left(v_{0}, \ldots, \hat{v}_{j}, \hat{v}_{i}, \ldots, v_{7}\right)} \otimes \\
& \frac{\triangle\left(v_{0}, \ldots, \hat{v}_{j+1}, \hat{v}_{i}, \ldots, v_{7}\right)}{\triangle\left(v_{0}, \ldots, \hat{v}_{j+2}, \hat{v}_{i}, \ldots, v_{7}\right)} \wedge \frac{\triangle\left(v_{0}, \ldots, \hat{v}_{j+2}, \hat{v}_{i}, \ldots, v_{7}\right)}{\triangle\left(v_{0}, \ldots, \hat{v}_{j+3}, \hat{v}_{i}, \ldots, v_{7}\right)} \wedge \\
& \frac{\triangle\left(v_{0}, \ldots, \hat{v}_{j+3}, \hat{v}_{i}, \ldots, v_{7}\right)}{\triangle\left(v_{0}, \ldots, \hat{v}_{j+4}, \hat{v}_{i}, \ldots, v_{7}\right)} \wedge \frac{\triangle\left(v_{0}, \ldots, \hat{v}_{j+4}, \hat{v}_{i}, \ldots, v_{7}\right)}{\triangle\left(v_{0}, \ldots, \hat{v}_{j+5}, \hat{v}_{i}, \ldots, v_{7}\right)} \wedge \\
& \frac{\triangle\left(v_{0}, \ldots, \hat{v}_{j+5}, \hat{v}_{i}, \ldots, v_{7}\right)}{\triangle\left(v_{0}, \ldots, \hat{v}_{j+6}, \hat{v}_{i}, \ldots, v_{7}\right)}+ \\
& \sum_{j=0}^{7-i}(-1)^{j+1} \sum_{i=0}^{7}(-1)^{i} \bigwedge_{\substack{j \neq i \\
j=0}}^{7} \frac{\triangle\left(v_{0}^{*}, \ldots, \hat{v}^{*}{ }_{j}, \hat{v}^{*}{ }_{i}, \ldots, v_{7}^{*}\right) \varepsilon}{\triangle\left(v_{0}, \ldots, \hat{v}_{j}, \hat{v}_{i}, \ldots, v_{7}\right) .}
\end{aligned}
$$

From Eq.(3.20) and Eq.(3.22), we must say that $g_{0 \varepsilon}^{6} \circ d=\delta_{\varepsilon} \circ g_{1 \varepsilon}^{6}$. 
3.3. Geometry for any Weight n. Using previous lemmas, work and calculation, we have constructed the following generalized commutative diagram

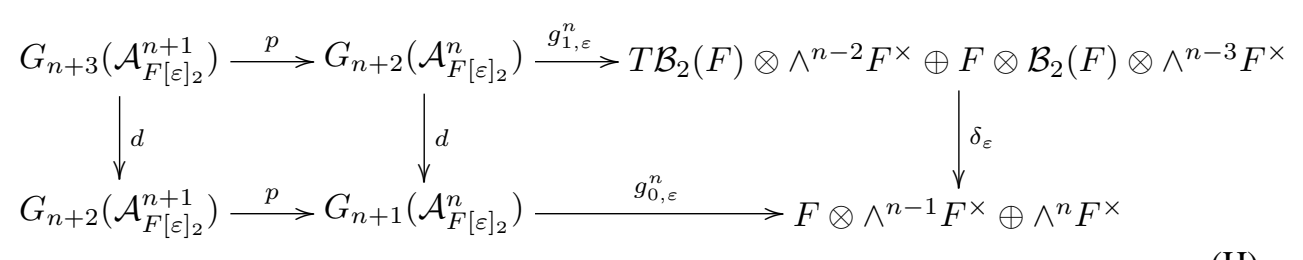

where, $g_{0 \varepsilon}^{n}\left(v_{0}^{*}, \ldots, v_{n}^{*}\right)=g_{0_{1}}^{n}\left(v_{0}^{*}, \ldots, v_{n}^{*}\right)+g_{0_{2}}^{n}\left(v_{0}^{*}, \ldots, v_{n}^{*}\right)$

$$
\begin{aligned}
& g_{0_{1}}^{n}\left(v_{0}^{*}, \ldots, v_{n}^{*}\right)=\sum_{i=j+1}^{n}(-1)^{i+1} \frac{\triangle\left(v_{0}^{*}, \ldots, \hat{v}^{*}, \ldots, v_{n}^{*}\right) \varepsilon}{\triangle\left(v_{0}, \ldots, \hat{v}_{i}, \ldots, v_{n}\right)} \otimes \frac{\triangle\left(v_{0}, \ldots, \hat{v}_{i+1}, \ldots, v_{n}\right)}{\triangle\left(v_{0}, \ldots, \hat{v}_{i+2}, \ldots, v_{n}\right)} \wedge \\
& \frac{\triangle\left(v_{0}, \ldots, \hat{v}_{i+2}, \ldots, v_{n}\right)}{\triangle\left(v_{0}, \ldots, \hat{v}_{i+3}, \ldots, v_{n}\right)} \wedge \frac{\triangle\left(v_{0}, \ldots, \hat{v}_{i+3}, \ldots, v_{n}\right)}{\triangle\left(v_{0}, \ldots, \hat{v}_{i+4}, \ldots, v_{n}\right)} \wedge \ldots \wedge \\
& \frac{\triangle\left(v_{0}, \ldots, \hat{v}_{i+n-1}, \ldots, v_{n}\right)}{\triangle\left(v_{0}, \ldots, \hat{v}_{i+n}, \ldots, v_{n}\right)}(i \bmod n+1), \\
& g_{0_{2}}^{n}\left(v_{0}^{*}, \ldots, v_{n}^{*}\right)=\sum_{j=0}^{n}(-1)^{j+1} \bigwedge_{\substack{j \neq i \\
j=0}}^{n} \frac{\triangle\left(v_{0}^{*}, \ldots, \hat{v}^{*}{ }_{j}, \ldots, v_{n}^{*}\right) \varepsilon}{\triangle\left(v_{0}, \ldots, \hat{v}_{j}, \ldots, v_{n}\right)}(i \bmod n+1)
\end{aligned}
$$

and

$$
\begin{aligned}
& g_{1 \varepsilon}^{n}\left(v_{0}^{*}, \ldots, v_{n+1}^{*}\right)=\frac{(-1)^{n+1}}{n+1} \sum_{2} \sum_{i_{0} \neq i_{1} \ldots \neq i_{n-2}}^{n+1}(-1)^{i}\left(\left\langler \left(v_{i_{0}}, v_{i_{1}}, \ldots, v_{i_{n-2}} \mid v_{0}, \ldots, \hat{v}_{i_{0}}, \hat{v}_{i_{1}}, \ldots, \hat{v}_{i_{n-2}}, \ldots,\right.\right.\right. \\
& \left.\left.v_{n+1}\right) ; r_{\varepsilon}\left(v_{i_{0}}, v_{i_{1}}, \ldots, v_{i_{n-2}} \mid v_{0}^{*}, \ldots, \hat{v}^{*}{ }_{i_{0}}, \hat{v}^{*}{ }_{i_{1}}, \ldots, \hat{v}^{*}{ }_{i_{n-2}}, \ldots, v_{n+1}^{*}\right)\right]_{2} \otimes \\
& \prod_{i_{0} \neq i_{0}+1 \neq \ldots \neq i_{0}+n-3}^{n+1} \triangle\left(v_{0}, \ldots, \hat{v}_{i_{0}}, \hat{v}_{i_{0}+1}, \ldots, \hat{v}_{i_{0}+n-3}, \ldots, v_{n+1}\right) \wedge \\
& \prod_{i_{1} \neq i_{1}+1}^{n+1} \triangle\left(v_{0}, \ldots, \hat{v}_{i_{1}}, \hat{v}_{i_{1}+1}, \ldots, \hat{v}_{i_{1}+n-3}, \ldots, v_{n+1}\right) \wedge \ldots \wedge \\
& \imath_{1} \neq i_{1}+1 \neq \ldots \neq i_{1}+n-3 \\
& \prod_{i_{n-2} \neq i_{n-2}+1 \neq \ldots \neq i_{n-2}+n-3}^{n+1} \triangle\left(v_{0}, \ldots, \hat{v}_{i_{n-2}}, \hat{v}_{i_{n-2}+1}, \ldots, \hat{v}_{i_{n-2}+n-3}, \ldots, v_{n+1}\right)+ \\
& \sum_{i_{0} \neq i_{0}+1 \neq \ldots \neq i_{0}+n}^{n+1} \frac{\triangle\left(v_{0}^{*}, \ldots, \hat{v}^{*}, \ldots, \hat{v}_{i_{0}}, \hat{v}^{*}{ }_{i_{0}+1}, \hat{v}_{i_{0}+1}, \hat{v}_{i_{0}+n-3}, \ldots, v_{n+1}\right)}{\left.\triangle v_{i_{0}}, \ldots, v_{n+1}^{*}\right) \varepsilon} \otimes \\
& {\left[v_{i_{0}}, v_{i_{1}}, \ldots, v_{i_{n-2}} \mid v_{0}^{*}, \ldots, \hat{v}^{*}{ }_{i_{0}},{\hat{v^{*}}}_{i_{1}}, \ldots,{\hat{v^{*}}}_{i_{n-2}}, \ldots, v_{n+1}^{*}\right]_{2} \otimes} \\
& \prod_{i_{1} \neq i_{1}+1 \neq \ldots \neq i_{1}+n-3}^{n+1} \triangle\left(v_{0}, \ldots, \hat{v}_{i_{1}}, \hat{v}_{i_{1}+1}, \ldots, \hat{v}_{i_{1}+n-3}, \ldots, v_{n+1}\right) \wedge \ldots \wedge
\end{aligned}
$$




$$
\begin{aligned}
& \prod_{i_{n-2} \neq i_{n-2}+1 \neq \ldots \neq i_{n-2}+n-3}^{n+1} \triangle\left(v_{0}, \ldots, \hat{v}_{i_{n-2}}, \hat{v}_{i_{n-2}+1}, \ldots, \hat{v}_{i_{n-2}+n-3}, \ldots, v_{n+1}\right)+ \\
& \sum_{i_{1} \neq i_{1+1} \neq \ldots \neq i_{1}+n}^{n+1} \frac{\triangle\left(v_{1}^{*}, \ldots, \hat{v}^{*}{ }_{i_{1}}, \hat{v}^{*}{ }_{i_{1}+1}, \hat{v}_{i_{1}}, \hat{v}_{i_{1}+1}, \hat{v}_{i_{1}+n-3}, \ldots, v_{n+1}\right)}{\triangle\left(v_{n+1}\right) \varepsilon} \\
& {\left[v_{i_{0}}, v_{i_{1}}, \ldots, v_{i_{n-2}} \mid v_{0}^{*}, \ldots, \hat{v}^{*} i_{0}, \hat{v}^{*}{ }_{i_{1}}, \ldots, \hat{v}^{*}{ }_{i_{n-2}}, \ldots, v_{n+1}^{*}\right]_{2} \otimes} \\
& \prod_{i_{0} \neq i_{0}+1 \neq \ldots \neq i_{0}+n-3}^{n+1} \triangle\left(v_{0}, \ldots, \hat{v}_{i_{0}}, \hat{v}_{i_{0}+1}, \ldots, \hat{v}_{i_{0}+n-3}, \ldots, v_{n+1}\right) \wedge \ldots \wedge \\
& \prod_{i_{n-2} \neq i_{n-2}+1 \neq \ldots \neq i_{n-2}+n-3}^{n+1} \triangle\left(v_{0}, \ldots, \hat{v}_{i_{n-2}}, \hat{v}_{i_{n-2}+1}, \ldots, \hat{v}_{i_{n-2}+n-3}, \ldots, v_{n+1}\right)+\cdots+ \\
& \sum_{i_{n-2} \neq i_{n-2}+1 \neq \ldots \neq i_{n-2}+n-3}^{n+1} \frac{\triangle\left(v_{1}^{*}, \ldots, \hat{v}^{*}{ }_{i_{n-2}}, \hat{v}^{*}{ }_{i_{n-2}+1}, \hat{v}_{i_{n-2}}, \hat{v}_{i_{n-2}+n-3}, \ldots, v_{n+1}^{*}\right) \varepsilon}{\triangle\left(v_{i_{n-2}+n-3}, \ldots, v_{n+1}\right)} \otimes \\
& {\left[v_{i_{0}}, v_{i_{1}}, \ldots, v_{i_{n-2}} \mid v_{0}^{*}, \ldots, \hat{v}^{*}{ }_{i_{0}}, \hat{v}^{*}{ }_{i_{1}}, \ldots, \hat{v}^{*}{ }_{i_{n-2}}, \ldots, v_{n+1}^{*}\right]_{2} \otimes} \\
& \prod_{i_{0} \neq i_{0}+1 \neq \ldots \neq i_{0}+n-3}^{n+1} \triangle\left(v_{0}, \ldots, \hat{v}_{i_{0}}, \hat{v}_{i_{0}+1}, \ldots, \hat{v}_{i_{0}+n-3}, \ldots, v_{n+1}\right) \wedge \ldots \wedge \\
& \prod_{i_{n-3} \neq i_{n-3}+1 \neq \ldots \neq i_{n-3}+n-3}^{n+1} \triangle\left(v_{0}, \ldots, \hat{v}_{i_{n-3}}, \hat{v}_{i_{n-3}+1}, \ldots, \hat{v}_{i_{n-3}+n-3}, \ldots, v_{n+1}\right) \\
& (\bmod n+2) \text {. }
\end{aligned}
$$

Theorem 3.4. $\quad g_{0 \varepsilon}^{n} \circ d=\delta_{\varepsilon} \circ g_{1 \varepsilon}^{n}$

Proof. Let $\left(v_{0}^{*}, \ldots, v_{n+1}^{*}\right)$ be $(n+2)$ points in space $G_{n+2}\left(\mathcal{A}_{F[\varepsilon]_{2}}^{n}\right)$ and apply morphism $d$, then we get

$$
d\left(v_{0}^{*}, \ldots, v_{n+1}^{*}\right)=\sum_{i=0}^{n+1}(-1)^{i}\left(v_{0}^{*}, \ldots, \hat{v}_{i}, \ldots, v_{n+1}^{*}\right)
$$

Now apply morphism $g_{0_{1}}^{n}$ then

$$
\begin{aligned}
& g_{0_{1}}^{n} \circ d\left(v_{0}^{*}, \ldots, v_{n+1}^{*}\right)=\sum_{j=i+1}^{n+1-i}(-1)^{j} \sum_{i=0}^{n+1}(-1)^{i} \frac{\triangle\left(v_{0}^{*}, \ldots, \hat{v}^{*}{ }_{j}, \hat{v}^{*}{ }_{i}, \ldots, v_{n+1}^{*}\right) \varepsilon}{\triangle\left(v_{0}, \ldots, \hat{v}_{j}, \hat{v}_{i}, \ldots, v_{n+1}\right)} \otimes \\
& \frac{\triangle\left(v_{0}, \ldots, \hat{v}_{j+1}, \hat{v}_{i}, \ldots, v_{n+1}\right)}{\triangle\left(v_{0}, \ldots, \hat{v}_{j+2}, \hat{v}_{i}, \ldots, v_{n+1}\right)} \wedge \frac{\triangle\left(v_{0}, \ldots, \hat{v}_{j+2}, \hat{v}_{i}, \ldots, v_{n+1}\right)}{\triangle\left(v_{0}, \ldots, \hat{v}_{j+3}, \hat{v}_{i}, \ldots, v_{n+1}\right)} \wedge \\
& \frac{\triangle\left(v_{0}, \ldots, \hat{v}_{j+3}, \hat{v}_{i}, \ldots, v_{n+1}\right)}{\triangle\left(v_{0}, \ldots, \hat{v}_{j+4}, \hat{v}_{i}, \ldots, v_{n+1}\right)} \wedge \ldots \wedge \frac{\triangle\left(v_{0}, \ldots, \hat{v}_{j+n-1}, \hat{v}_{i}, \ldots, v_{n+1}\right)}{\triangle\left(v_{0}, \ldots, \hat{v}_{j+n}, \hat{v}_{i}, \ldots, v_{n+1}\right) .}
\end{aligned}
$$


Now compose morphism $g_{0_{2}}^{n}$ and $d\left(v_{0}^{*}, \ldots, v_{n+1}^{*}\right)$ then

$$
g_{0_{2}}^{n} \circ d\left(v_{0}^{*}, \ldots, v_{n+1}^{*}\right)=\sum_{j=i+1}^{n+1-i}(-1)^{j} \sum_{i=0}^{n+1}(-1)^{i} \bigwedge_{\substack{j \neq i \\ j=0}}^{n} \frac{\triangle\left(v_{0}^{*}, \ldots, \hat{v}^{*}{ }_{j}, \ldots, v_{n}^{*}\right) \varepsilon}{\triangle\left(v_{0}, \ldots, \hat{v}_{j}, \ldots, v_{n}\right) .}
$$

The morphism $g_{0 \varepsilon}^{n}=g_{0_{1}}^{n}+g_{0_{2}}^{n}$, therefore combine Eq.(3.26) and Eq.(3.27), it follows

$$
\begin{aligned}
g_{0 \varepsilon}^{n} \circ d\left(v_{0}^{*}, \ldots, v_{n+1}^{*}\right) & =\sum_{j=i+1}^{n+1-i}(-1)^{j} \sum_{i=0}^{n+1}(-1)^{i} \frac{\triangle\left(v_{0}^{*}, \ldots, \hat{v}^{*}, \hat{v}^{*}{ }_{i}, \ldots, v_{n+1}^{*}\right) \varepsilon}{\triangle\left(v_{0}, \ldots, \hat{v}_{j}, \hat{v}_{i}, \ldots, v_{n+1}\right)} \otimes \\
& \frac{\triangle\left(v_{0}, \ldots, \hat{v}_{j+1}, \hat{v}_{i}, \ldots, v_{n+1}\right)}{\triangle\left(v_{0}, \ldots, \hat{v}_{j+2}, \hat{v}_{i}, \ldots, v_{n+1}\right)} \wedge \frac{\triangle\left(v_{0}, \ldots, \hat{v}_{j+2}, \hat{v}_{i}, \ldots, v_{n+1}\right)}{\triangle\left(v_{0}, \ldots, \hat{v}_{j+3}, \hat{v}_{i}, \ldots, v_{n+1}\right)} \wedge \\
& \frac{\triangle\left(v_{0}, \ldots, \hat{v}_{j+3}, \hat{v}_{i}, \ldots, v_{n+1}\right)}{\triangle\left(v_{0}, \ldots, \hat{v}_{j+4}, \hat{v}_{i}, \ldots, v_{n+1}\right)} \wedge \ldots \frac{\triangle\left(v_{0}, \ldots, \hat{v}_{j+n-1}, \hat{v}_{i}, \ldots, v_{n+1}\right)}{\triangle\left(v_{0}, \ldots, \hat{v}_{j+n}, \hat{v}_{i}, \ldots, v_{n+1}\right)}+ \\
& \sum_{j=i+1}^{n+1-i}(-1)^{j} \sum_{i=0}^{n+1}(-1)^{i} \bigwedge_{\substack{j \neq i \\
j=0}}^{n} \frac{\triangle\left(v_{0}^{*}, \ldots, \hat{v}^{*}{ }_{j}, \ldots, v_{n}^{*}\right) \varepsilon}{\triangle\left(v_{0}, \ldots, \hat{v}_{j}, \ldots, v_{n}\right) .}
\end{aligned}
$$

Take again $\left(v_{0}^{*}, \ldots, v_{n+1}^{*}\right) \in G_{n+2}\left(\mathcal{A}_{F[\varepsilon]_{2}}^{n}\right)$ and apply morphism $g_{1 \varepsilon}^{n}$, it follows

$$
\begin{aligned}
& g_{1 \varepsilon}^{n}\left(v_{0}^{*}, \ldots, v_{n+1}^{*}\right)=\frac{(-1)^{n+1}}{n+1 C_{2}} \sum_{i_{0} \neq i_{1} \ldots \neq i_{n-2}}^{n+1}(-1)^{i}\left(\left\langler \left(v_{i_{0}}, v_{i_{1}}, \ldots, v_{i_{n-2}} \mid v_{0}, \ldots, \hat{v}_{i_{0}},\right.\right.\right. \\
& \left.\hat{v}_{i_{1}}, \ldots, \hat{v}_{i_{n-2}}, \ldots, v_{n+1}\right) ; r_{\varepsilon}\left(v_{i_{0}}, v_{i_{1}}, \ldots, v_{i_{n-2}} \mid v_{0}^{*}, \ldots,\right. \\
& \left.\left.\hat{v}^{*}{ }_{i_{0}}, \hat{v}^{*} i_{1}, \ldots, \hat{v}^{*}{ }_{i_{n-2}}, \ldots, v_{n+1}^{*}\right)\right]_{2} \otimes \\
& \prod_{i_{0} \neq i_{0}+1 \neq \ldots \neq i_{0}+n-3}^{n+1} \triangle\left(v_{0}, \ldots, \hat{v}_{i_{0}}, \hat{v}_{i_{0}+1}, \ldots, \hat{v}_{i_{0}+n-3}, \ldots, v_{n+1}\right) \wedge \\
& \prod_{i_{1} \neq i_{1}+1 \neq \ldots \neq i_{1}+n-3}^{n+1} \triangle\left(v_{0}, \ldots, \hat{v}_{i_{1}}, \hat{v}_{i_{1}+1}, \ldots, \hat{v}_{i_{1}+n-3}, \ldots, v_{n+1}\right) \wedge \ldots \wedge \\
& \prod_{i_{n-2} \neq i_{n-2}+1 \neq \ldots \neq i_{n-2}+n-3}^{n+1} \triangle\left(v_{0}, \ldots, \hat{v}_{i_{n-2}}, \hat{v}_{i_{n-2}+1}, \ldots, \hat{v}_{i_{n-2}+n-3}, \ldots, v_{n+1}\right)+ \\
& \sum_{i_{0} \neq i_{0}+1 \neq \ldots \neq i_{0}+n}^{n+1} \frac{\triangle\left(v_{0}^{*}, \ldots, \hat{v}^{*} i_{0}, \hat{v}^{*}{ }_{i_{0}+1}, \hat{v}^{*} i_{i_{0}}, \hat{v}_{i_{0}+1}, \hat{v}_{i_{0}+n-3}, \ldots, v_{n+1}\right)}{\triangle\left(v_{n+1}\right) \varepsilon} \otimes \\
& {\left[v_{i_{0}}, v_{i_{1}}, \ldots, v_{i_{n-2}} \mid v_{0}^{*}, \ldots, \hat{v}^{*}{ }_{i_{0}}, \hat{v}^{*}{ }_{i_{1}}, \ldots,{\hat{v^{*}}}_{i_{n-2}}, \ldots, v_{n+1}^{*}\right]_{2} \otimes} \\
& \prod_{i_{1} \neq i_{1}+1 \neq \ldots \neq i_{1}+n-3}^{n+1} \triangle\left(v_{0}, \ldots, \hat{v}_{i_{1}}, \hat{v}_{i_{1}+1}, \ldots, \hat{v}_{i_{1}+n-3}, \ldots, v_{n+1}\right) \wedge \ldots \wedge \\
& \prod_{i_{n-2}}^{n+1} \triangle\left(v_{0}, \ldots, \hat{v}_{i_{n-2}}, \hat{v}_{i_{n-2}+1}, \ldots, \hat{v}_{i_{n-2}+n-3}, \ldots, v_{n+1}\right)+
\end{aligned}
$$




$$
\begin{aligned}
& \sum_{\left.i_{1} \neq i_{1+1} \neq \ldots \neq i_{1}+n-3_{0}, \ldots, \hat{v}_{i_{1}}, \hat{v}_{i_{1}+1}, \hat{v}_{i_{1}+n-3}, \ldots, v_{n+1}\right)}^{n+1} \frac{\triangle\left(v_{1}^{*}, \ldots, \hat{v}^{*}{ }_{i_{1}}, \hat{v}^{*}{ }_{i_{1}+1}, \hat{v}^{*}{ }_{i_{1}+n-3}, \ldots, v_{n+1}^{*}\right) \varepsilon}{\triangle\left(v_{1}\right)} \\
& {\left[v_{i_{0}}, v_{i_{1}}, \ldots, v_{i_{n-2}} \mid v_{0}^{*}, \ldots, \hat{v}^{*} i_{0}, \hat{v}^{*}{ }_{i_{1}}, \ldots, \hat{v}^{*}{ }_{i_{n-2}}, \ldots, v_{n+1}^{*}\right]_{2} \otimes} \\
& \prod_{i_{0} \neq i_{0}+1 \neq \ldots \neq i_{0}+n-3}^{n+1} \triangle\left(v_{0}, \ldots, \hat{v}_{i_{0}}, \hat{v}_{i_{0}+1}, \ldots, \hat{v}_{i_{0}+n-3}, \ldots, v_{n+1}\right) \wedge \ldots \wedge \\
& \prod_{i_{n-2} \neq i_{n-2}+1 \neq \ldots \neq i_{n-2}+n-3}^{n+1} \triangle\left(v_{0}, \ldots, \hat{v}_{i_{n-2}}, \hat{v}_{i_{n-2}+1}, \ldots, \hat{v}_{i_{n-2}+n-3}, \ldots, v_{n+1}\right)+\cdots+ \\
& \sum_{\left.i_{n-2} \neq i_{n-2}+1 \neq \ldots \neq i_{n-2}+n \underline{-}_{3} \ldots, \hat{v}_{i_{n-2}}, \hat{v}_{i_{n-2}+1}, \hat{v}_{i_{n-2}+n-3}, \ldots, v_{n+1}\right)}^{\triangle\left(v_{1}^{*}, \ldots, \hat{v}^{*}{ }_{i_{n-2}}, \hat{v}^{*}{ }_{i_{n-2}+1}, \hat{v}_{i_{n-2}+n-3}, \ldots, v_{n+1}^{*}\right) \varepsilon} \otimes \\
& {\left[v_{i_{0}}, v_{i_{1}}, \ldots, v_{i_{n-2}} \mid v_{0}^{*}, \ldots, \hat{v}^{*}{ }_{i_{0}}, \hat{v}^{*}{ }_{i_{1}}, \ldots, \hat{v}^{*}{ }_{i_{n-2}}, \ldots, v_{n+1}^{*}\right]_{2} \otimes} \\
& \prod_{i_{0} \neq i_{0}+1 \neq \ldots \neq i_{0}+n-3}^{n+1} \triangle\left(v_{0}, \ldots, \hat{v}_{i_{0}}, \hat{v}_{i_{0}+1}, \ldots, \hat{v}_{i_{0}+n-3}, \ldots, v_{n+1}\right) \wedge \ldots \wedge \\
& \prod_{i_{n-3} \neq i_{n-3}+1 \neq \ldots \neq i_{n-3}+n-3}^{n+1} \triangle\left(v_{0}, \ldots, \hat{v}_{i_{n-3}}, \hat{v}_{i_{n-3}+1}, \ldots, \hat{v}_{i_{n-3}+n-3}, \ldots, v_{n+1}\right) .
\end{aligned}
$$

Now compose morphisms $\delta_{\varepsilon}$ and $g_{1 \varepsilon}^{n}\left(v_{0}^{*}, \ldots, v_{n+1}^{*}\right)$ and simplifying using tensor, cycle, Siegel cross ratio and wedge properties, the above equation becomes

$$
\begin{aligned}
\delta_{\varepsilon} \circ g_{1 \varepsilon}^{n}\left(v_{0}^{*}, \ldots, v_{n+1}^{*}\right) & =\sum_{j=i+1}^{n+1-i}(-1)^{j} \sum_{i=0}^{n+1}(-1)^{i} \frac{\triangle\left(v_{0}^{*}, \ldots, \hat{v}^{*}{ }_{j}, \hat{v}^{*}{ }_{i}, \ldots, v_{n+1}^{*}\right) \varepsilon}{\triangle\left(v_{0}, \ldots, \hat{v}_{j}, \hat{v}_{i}, \ldots, v_{n+1}\right)} \otimes \\
& \frac{\triangle\left(v_{0}, \ldots, \hat{v}_{j+1}, \hat{v}_{i}, \ldots, v_{n+1}\right)}{\triangle\left(v_{0}, \ldots, \hat{v}_{j+2}, \hat{v}_{i}, \ldots, v_{n+1}\right)} \wedge \frac{\triangle\left(v_{0}, \ldots, \hat{v}_{j+2}, \hat{v}_{i}, \ldots, v_{n+1}\right)}{\triangle\left(v_{0}, \ldots, \hat{v}_{j+3}, \hat{v}_{i}, \ldots, v_{n+1}\right)} \wedge \\
& \frac{\triangle\left(v_{0}, \ldots, \hat{v}_{j+3}, \hat{v}_{i}, \ldots, v_{n+1}\right)}{\triangle\left(v_{0}, \ldots, \hat{v}_{j+4}, \hat{v}_{i}, \ldots, v_{n+1}\right)} \wedge \ldots \wedge \frac{\triangle\left(v_{0}, \ldots, \hat{v}_{j+n-1}, \hat{v}_{i}, \ldots, v_{n+1}\right)}{\triangle\left(v_{0}, \ldots, \hat{v}_{j+n}, \hat{v}_{i}, \ldots, v_{n+1}\right)}+ \\
& \sum_{j=i+1}^{n+1-i}(-1)^{j} \sum_{i=0}^{n+1}(-1)^{i} \bigwedge_{j \neq i}^{n} \frac{\triangle\left(v_{0}^{*}, \ldots, \hat{v}^{*}{ }_{j}, \ldots, v_{n}^{*}\right) \varepsilon}{\triangle\left(v_{0}, \ldots, \hat{v}_{j}, \ldots, v_{n}\right) .}
\end{aligned}
$$

From Eq.(3.28) and Eq.(3.30), we must say that $g_{0 \varepsilon}^{n} \circ d=\delta_{\varepsilon} \circ g_{1 \varepsilon}^{n}$.

\section{CONCLUSion}

Generalized geometry between Tangent groups and affine configuration chain complexes is studied in detail. In the previous work, researchers discussed geometry only for lower weights but here generalized weight $n$ is proposed by some valuable generalized homomorphism. In past research, researchers defined only few arbitrary homomorphisms for weight $n=2$ up to weight $n=5$. But here we generalized all homomorphisms for any weight $n \in \mathbb{N}$. 


\section{REFERENCES}

[1] S. Bloch, Applications of the dilogarithm function in algebraic K-theory and algebraic geometry, Proc. int. Symp. on Algebraic Geometry (Kyoto Univ., Kyoto, Japan), pp. 1031141978.

[2] J.L. Cathelineau, Remarques sur les différentielles des polylogarithmes uniformes, Ann. Inst. Fourier, Grenoble, 46, No.5 (1996) 1327-1347. doi:10.5802/aif.1551

[3] J.L. Cathelineau, Infinitesimal polylogarithms, multiplicative presentation of Kähler Differential and Gonchrove complexes, Talk at the workshop on polylogarthms, Essen, Germany, May 1-4, 1997.

[4] J.L. Cathelineau, Infinitesimal polylogarithms, The Tangent complex to Bloch Suslin complex, Bull. Soc. math. France, 13,5 No.4 (2007) 565-597.

[5] J.L. Dupont and C.H. Sah, Homology of Euclidean groups of motion made discrete and Euclidean scissors congruences, Acta Math., 164 (1990) 1-24.

[6] A.B. Goncharov, The classical trilogarithm, algebraic K-theory of field and Dedekind zeta functions, Bull. of AMS., 24, No.1 (1991) 155-162.

[7] A.B. Goncharov, Geometry of configuration, polylogarithms and motivic cohomology, Adv. Math., 114, No.2 (1995) 197-318. doi:10.1006/aima.1995.1045.

[8] A.B. Goncharov, Geometry of the trilogarithm and the motivic Lie algebra of a field, Regulators in Analysis, Geometry and Number Theory, 171 (2000) 127-165.

[9] A.B. Goncharov and J. Zhao, Grassmannian trilogarithm, Compositio Mathematica, 127,No. 1 (2001) 93108. doi:10.1023/A:1017504115184.

[10] A.B. Goncharov, Euclidean scissor congruence groups and mixed Tate motives over dual numbers, Mathematical Research Letters, 11, No.6 (2004) 771-784. doi:10.4310/MRL.2004.v11.n6.a5.

[11] S. Hussain and R. Siddiqui, Grassmannian complex and second order tangent complex, Punjab Uni. j. of math. 48,No.2 (2016) 91-111.

[12] M. Khalid, J. Khan and A. Iqbal, New homomorphism between Grassmannian and infinitesimal complexes, International Journal of Algebra, 10, No.3 (2016) 97-112. doi:10.12988/ija.2016.6213.

[13] M. Khalid, J. Khan and A. Iqbal, Generalization of Grassmannian and polylogarithmic groups complexes, International Journal of Algebra, 10, No.5 (2016) 221-237. doi:10.12988/ija.2016.6323.

[14] M. Khalid, J. Khan and A. Iqbal, Higher order Grassmannian complexes, International Journal of Algebra, 10, No.9 (2016) 405-413. doi:10.12988/ija.2016.6640

[15] M. Khalid, J. Khan and A. Iqbal, Generalization of higher order homomorphism in configuration complexes, Punjab Univ. j. math. 49, No.2 (2017) 37-49.

[16] M. Khalid, A. Iqbal and J. Khan, Generalization of the geometry of Cathelineau infinitesimal and Grassmannian chain complexes, Preprint, 2017, doi10.20944/preprints201703.0098.v1

[17] M. Khalid, J. Khan and A. Iqbal, Generalized geometry of Goncharov and configuration complexes, Turk. J. Math., 42,No. 3 (2018) 1509-1527. doi:10.3906/mat-1702-25

[18] M. Khalid, J. Khan and A. Iqbal, Extension of morphisms in geometry of chain Complexes, Punjab Univ. j. math., 51, No.1 (2019) 29-49.

[19] M. Khalid and A. Iqbal, Generalized extension of morphisms in geometry of configuration and infinitesimal polylogarithmic groups complexes, Punjab Univ. j. math., 51, No. 8 (2019) 111-127.

[20] M. Khalid, M. Sultana, J. Khan and A. Iqbal, Geometry of Tangential and configuration chain complexes for higher weights, Punjab Univ. j. bath., 52, No.2 (2020) 29-55.

[21] R.Siddiqui, Configuration complexes and tangential and infinitesimal version of polylogarithmic complexes, Doctoral thesis, Durham University, 2010.

[22] C.L. Siegel, Approximation algebraischer zahlen, Mathem.Ze/tschr, 10 (1921) 173-213. (in German)

[23] A.A. Suslin, Homology of $G L_{n}$, characteristic classes and Milnor's K-theory, In Proceedings of the Steklov Institute of Mathematics 1985, Lecture Notes in Mathematics (1046), New York, USA, Springer-Verlag 1989; pp. 207-226. 